\title{
Fixed point solutions of generalized mixed equilibrium problems and variational inclusion problems for nonexpansive semigroups
}

Jae Ug Jeong*

\section{"Correspondence:} jujeong@deu.ac.kr Department of Mathematics, Dongeui University, Busan, 614-714, South Korea

\section{Springer}

\begin{abstract}
In this paper, we introduce a composite iterative method for solving a common element of the set of solutions of fixed points for nonexpansive semigroups, the set of solutions of generalized mixed equilibrium problems and the set of solutions of the variational inclusion for a $\beta$-inverse strongly monotone mapping in a real Hilbert space. We prove that the sequence converges strongly to a common element of the above sets under some mild conditions. Our results improve and extend the corresponding results of Kumam and Wattanawitoon (Math. Comput. Model. 53:998-1006, 2011), Shehu (Math. Comput. Model. 55:1301-1314, 2012), Plubtieng and Punpaeng (Math. Comput. Model. 48:279-286, 2008), Li et al. (Nonlinear Anal. 70:3065-3071, 2009), Plubtieng and Wangkeeree (Bull. Korean Math. Soc. 45:717-728, 2008) and some authors.

MSC: $46 \mathrm{CO} 5 ; 47 \mathrm{H09} ; 47 \mathrm{H} 10$
\end{abstract}

Keywords: variational inequality; inverse strongly monotone mapping; generalized mixed equilibrium problem; nonexpansive semigroup

\section{Introduction}

Let $H$ be a real Hilbert space with inner product $\langle\cdot, \cdot\rangle$ and norm $\|\cdot\|$. Let $C$ be a nonempty closed convex subset of $H$. Recall that a mapping $T: C \rightarrow C$ is said to be nonexpansive if

$$
\|T x-T y\| \leq\|x-y\|, \quad \forall x, y \in C .
$$

We denote by $F(T)$ the set of fixed points of $T$. Let $P_{C}$ be the projection of $H$ onto the convex subset $C$. Moreover, we also denote by $\mathbb{R}$ the set of all real numbers.

A one-parameter family $\mathfrak{s}=\{T(t): 0 \leq t<\infty\}$ is said to be a nonexpansive semigroup on $C$ if it satisfies the following conditions:

(1) $T(0) x=x$ for all $x \in C$;

(2) $T(s+t)=T(s) T(t)$ for all $s, t \geq 0$;

(3) $\|T(t) x-T(t) y\| \leq\|x-y\|$ for all $x, y \in C$ and $t \geq 0$;

(4) for all $x \in C, t \mapsto T(t) x$ is continuous.

We denote by $F(\Im)$ the set of all common fixed points of $\Im$, that is, $F(\Im)=\{x \in C: T(t) x=$ $x, 0 \leq t<\infty\}$. It is well known that $F(\Im)$ is closed and convex.

○2014 Jeong; licensee Springer. This is an Open Access article distributed under the terms of the Creative Commons Attribution License (http://creativecommons.org/licenses/by/2.0), which permits unrestricted use, distribution, and reproduction in any medium, provided the original work is properly cited. 
A mapping $A$ of $C$ into $H$ is called monotone if $\langle A x-A y, x-y\rangle \geq 0$ for all $x, y \in C$. A mapping $A$ is called $\alpha$-inverse strongly monotone if there exists a positive real number $\alpha$ such that $\langle A x-A y, x-y\rangle \geq \alpha\|A x-A y\|^{2}$ for all $x, y \in C$. A mapping $A$ is called $\alpha$-strongly monotone if there exists a positive real number $\alpha$ such that $\langle A x-A y, x-y\rangle \geq \alpha\|x-y\|^{2}$ for all $x, y \in C$. It is obvious that any $\alpha$-inverse strongly monotone mappings $A$ is a monotone and $\frac{1}{\alpha}$-Lipschitz continuous mapping. A linear bounded operator $A$ is called strongly positive if there exists a constant $\bar{\gamma}>0$ with the property $\langle A x, x\rangle \geq \bar{\gamma}\|x\|^{2}$ for all $x \in H$.

Let $B: H \rightarrow H$ be a single-valued nonlinear mapping and $M: H \rightarrow 2^{H}$ be a set-valued mapping. The variational inclusion problem is to find $x \in H$ such that

$$
0 \in B(x)+M(x)
$$

where 0 is the zero vector in $H$.

The set of solutions of (1.1) is denoted by $I(B, M)$ (see [1-3] and the reference therein).

A set-valued mapping $M: H \rightarrow 2^{H}$ is called monotone if for all $x, y \in H, f \in M(x)$ and $g \in M(y)$ imply $\langle x-y, f-g\rangle \geq 0$. A monotone mapping $M$ is maximal if its graph $G(M)=\{(f, x) \in H \times H: f \in M(x)\}$ of $M$ is not properly contained in the graph of any other monotone mapping. It is well known that a monotone mapping $M$ is maximal if and only if for $(x, f) \in H \times H,\langle x-y, f-g\rangle \geq 0$ for all $(y, g) \in G(M)$ imply $f \in M(x)$.

Let $M: H \rightarrow 2^{H}$ be a set-valued maximal monotone mapping. Then the single-valued mapping $J_{M, \lambda}: H \rightarrow H$ defined by

$$
J_{M, \lambda}(x)=(I+\lambda M)^{-1}(x), \quad x \in H,
$$

is called the resolvent operator associated with $M$, where $\lambda$ is any positive number and $I$ is the identity mapping. It is well known that the resolvent operator $J_{M, \lambda}$ is nonexpansive, 1-inverse strongly monotone and that a solution of problem (1.1) is a fixed point of the operator $J_{M, \lambda}(I-\lambda B)$ for all $\lambda>0$, where $I$ denotes the identity operator on $H$ (see [4]).

Peng and Yao [5] considered the following generalized mixed equilibrium problem of finding $x \in C$ such that

$$
\theta(x, y)+\varphi(y)-\varphi(x)+\langle F x, y-x\rangle \geq 0, \quad \forall y \in C,
$$

where $F: C \rightarrow H$ is a nonlinear mapping, $\varphi: C \rightarrow \mathbb{R}$ is a function and $\theta: C \times C \rightarrow \mathbb{R}$ is a bifunction. The set of solutions of problem (1.2) is denoted by GMEP.

In the case of $F=0$, problem (1.2) reduces to the following mixed equilibrium problem of finding $x \in C$ such that

$$
\theta(x, y)+\varphi(y)-\varphi(x) \geq 0, \quad \forall y \in C,
$$

which was considered by Ceng and Yao [6]. GMEP is denoted by MEP.

In the case of $\varphi=0$, problem (1.2) reduces to the following generalized equilibrium problem of finding $x \in C$ such that

$$
\theta(x, y)+\langle F x, y-x\rangle \geq 0, \quad \forall y \in C,
$$

which was studied by Takahashi and Takahashi [7]. 
In the case of $\varphi=0$ and $F=0$, problem (1.2) reduces to the equilibrium problem of finding $x \in C$ such that

$$
\theta(x, y) \geq 0, \quad \forall y \in C
$$

The set of solution of (1.3) is denoted by $E P(\theta)$.

In the case $\theta=0$ and $\varphi=0$, problem (1.2) reduces to the classical variational inequality of finding $x \in C$ such that

$$
\langle F x, y-x\rangle \geq 0, \quad \forall y \in C
$$

The set of solutions of problem (1.4) is denoted by $\operatorname{VI}(F, C)$.

The problem (1.2) is very general in the sense that it includes, as special cases, optimization problem, variational inequalities, minimax problems, the Nash equilibrium problem in noncooperative games and others (see [6, 8-10]).

Peng and Yao [5] considered iterative methods for finding a common element of the set of solutions of problem (1.2), the set of solutions of problem (1.4), and the set of fixed points of a nonexpansive mapping.

Let $G_{1}, G_{2}: C \times C \rightarrow \mathbb{R}$ be two bifunctions, $\phi, \psi: C \rightarrow \mathbb{R}$ be two functions and $B_{1}, B_{2}$ : $C \rightarrow H$ be two nonlinear mappings. We consider the generalized mixed equilibria problem of finding $(\bar{x}, \bar{y}) \in C \times C$ such that

$$
\begin{cases}G_{1}(\bar{x}, x)+\phi(x)-\phi(\bar{x})+\left\langle B_{1} \bar{y}, x-\bar{x}\right\rangle+\frac{1}{\mu_{1}}\langle\bar{x}-\bar{y}, x-\bar{x}\rangle \geq 0, & \forall x \in C \\ G_{2}(\bar{y}, y)+\psi(y)-\psi(\bar{y})+\left\langle B_{2} \bar{x}, y-\bar{y}\right\rangle+\frac{1}{\mu_{2}}\langle\bar{y}-\bar{x}, y-\bar{y}\rangle \geq 0, & \forall y \in C\end{cases}
$$

where $\mu_{1}>0$ and $\mu_{2}>0$ are two constants.

In the case $\phi=\psi=0$, problem (1.5) reduces to the following problem of the general system of generalized equilibria of finding $(\bar{x}, \bar{y}) \in C \times C$ such that

$$
\begin{cases}G_{1}(\bar{x}, x)+\left\langle B_{1} \bar{y}, x-\bar{x}\right\rangle+\frac{1}{\mu_{1}}\langle\bar{x}-\bar{y}, x-\bar{x}\rangle \geq 0, & \forall x \in C, \\ G_{2}(\bar{y}, y)+\left\langle B_{2} \bar{x}, y-\bar{y}\right\rangle+\frac{1}{\mu_{2}}\langle\bar{y}-\bar{x}, y-\bar{y}\rangle \geq 0, & \forall y \in C,\end{cases}
$$

which was considered by Ceng and Yao [9].

In the case $G_{1}=G_{2}=0$, problem (1.6) reduces to the following problem of the general system of variational inequalities of finding $(\bar{x}, \bar{y}) \in C \times C$ such that

$$
\begin{cases}\left\langle\mu_{1} B_{1} \bar{y}+\bar{x}-\bar{y}, x-\bar{x}\right\rangle \geq 0, & \forall x \in C \\ \left\langle\mu_{2} B_{2} \bar{x}+\bar{y}-\bar{x}, y-\bar{y}\right\rangle \geq 0, & \forall y \in C\end{cases}
$$

which was considered by Ceng, Wang and Yao [11].

In particular, if $B_{1}=B_{2}=D: C \rightarrow H$ is a nonlinear mapping, then problem (1.7) reduces to the following problem of the system of variational inequalities of finding $(\bar{x}, \bar{y}) \in C \times C$ 
such that

$$
\begin{cases}\left\langle\mu_{1} D \bar{y}+\bar{x}-\bar{y}, x-\bar{x}\right\rangle \geq 0, & \forall x \in C, \\ \left\langle\mu_{2} D \bar{x}+\bar{y}-\bar{x}, y-\bar{y}\right\rangle \geq 0, & \forall y \in C,\end{cases}
$$

which was studied by Verma [12].

If $\bar{x}=\bar{y}$ in (1.8), then (1.8) reduces to the classical variational inequality (1.4).

For solving the mixed equilibrium problem, let us give the following assumptions for the bifunction $\theta, \varphi$ and the set $C$ :

(H1) $\theta(x, y)=0$ for all $x \in C$;

(H2) $\theta$ is monotone, i.e., $\theta(x, y)+\theta(y, x) \leq 0$ for all $x, y \in C$;

(H3) for each $x, y, z \in C$

$$
\limsup _{t \rightarrow 0} \theta(t z+(1-t) x, y) \leq \theta(x, y)
$$

(H4) for each $x \in C, y \mapsto \theta(x, y)$ is convex and lower semicontinuous;

(A1) for each $x \in H$ and $r>0$, there exist a bounded subset $D \subset C$ and $y \in C$ such that for any $z \in C \backslash D$

$$
\theta(z, y)+\varphi(y)-\varphi(z)+\frac{1}{r}\langle y-z, z-x\rangle<0
$$

(A2) $C$ is a bounded set.

Recently, Shehu [13] studied the problem of finding a common element of the set of common fixed points of a one-parameter nonexpansive semigroup, the set of solutions to a variational inclusion, and the set of solutions to a generalized equilibrium problem. More precisely, the author proved the following theorem.

Theorem 1.1 Let C be a nonempty closed and convex subset of a real Hilbert space H. Let $\theta$ be a bifunction from $C \times C$ to $\mathbb{R}$ satisfying $(\mathrm{H} 1)-(\mathrm{H} 4), \psi$ a $\mu$-inverse strongly monotone mapping of $C$ into $H, B$ an $\alpha$-inverse strongly monotone mapping of $C$ into $H$ and $M$ : $H \rightarrow 2^{H}$ a maximal monotone mapping. Let $\Im=\{T(u): 0 \leq u<\infty\}$ be a one-parameter nonexpansive semigroup on $H$ such that $\mathcal{F}=F(\Im) \cap I(B, M) \cap E P(\theta) \neq \phi$ and suppose $f$ : $H \rightarrow H$ is a contraction mapping with a constant $\gamma \in(0,1)$. Let $\left\{t_{n}\right\} \subset(0, \infty)$ be a real sequence such that $\lim _{n \rightarrow \infty} t_{n}=\infty$. Suppose $\left\{x_{n}\right\}_{n=1}^{\infty}$ and $\left\{u_{n}\right\}_{n=1}^{\infty}$ are generated by $x_{1} \in H$,

$$
\begin{aligned}
& \theta\left(u_{n}, y\right)+\left\langle\psi x_{n}, y-u_{n}\right\rangle+\frac{1}{r_{n}}\left\langle y-u_{n}, u_{n}-x_{n}\right\rangle \geq 0, \quad \forall y \in C, \\
& x_{n+1}=\beta_{n} x_{n}+\left(1-\beta_{n}\right)\left(\frac{1}{t_{n}} \int_{0}^{t_{n}} T(u)\left[\alpha_{n} f\left(x_{n}\right)+\left(1-\alpha_{n}\right) J_{M, \lambda}\left(u_{n}-\lambda A u_{n}\right)\right] d u\right)
\end{aligned}
$$

for all $n \geq 1$, where $\left\{\alpha_{n}\right\}_{n=1}^{\infty}$ and $\left\{\beta_{n}\right\}_{n=1}^{\infty}$ are sequences in $(0,1)$ and $\left\{r_{n}\right\}_{n=1}^{\infty} \subset(0, \infty)$ satisfying:

(i) $\lim _{n \rightarrow \infty} \beta_{n}=0, \sum_{n=1}^{\infty}\left|\beta_{n+1}-\beta_{n}\right|<\infty$,

(ii) $\lim _{n \rightarrow \infty} \alpha_{n}=0, \sum_{n=1}^{\infty} \alpha_{n}=\infty, \sum_{n=1}^{\infty}\left|\alpha_{n+1}-\alpha_{n}\right|<\infty$,

(iii) $\lambda \in(0,2 \alpha]$, 
(iv) $0<a \leq r_{n} \leq b<2 \mu, \sum_{n=1}^{\infty}\left|r_{n+1}-r_{n}\right|<\infty$,

(v) $\lim _{n \rightarrow \infty} \frac{\left|t_{n}-t_{n-1}\right|}{t_{n}} \frac{1}{\alpha_{n}\left(1-\beta_{n}\right)}=0$.

Then $\left\{x_{n}\right\}_{n=1}^{\infty}$ converges strongly to $z$, where $z=P_{\mathcal{F}} f(z)$.

In this paper, motivated by Shehu [13], Kumam and Wattanawitoon [14], Li et al. [15], Plubtieng and Punpaeng [10], Plubtieng and Wangkeeree [16], we introduce the following general iterative scheme for finding a common element of the set of common fixed points of a one-parameter nonexpansive semigroup, the set of solutions of the generalized mixed equilibrium problem (1.2), the set of solutions to a variational inclusion (1.1), and the set of solutions of the generalized mixed equilibria problem (1.5), which solves the variational inequality

$$
\left\langle(A-\gamma f) x^{*}, x-x^{*}\right\rangle \geq 0, \quad \forall x \in \mathcal{F},
$$

where $\mathcal{F}=F(\Im) \cap G M E P \cap \Omega \cap I(B, M)$ and $\Omega$ is the set of solutions of the generalized equilibria problem (1.5).

The results obtained in this paper improve and extend the recent results announced by $[10,13-16]$ and many others.

\section{Preliminaries}

Let $C$ be a nonempty closed convex subset of a real Hilbert space $H$. For every point $x \in H$ there exists a unique nearest point of $C$, denoted by $P_{C} x$ such that $\left\|x-P_{C} x\right\| \leq\|x-y\|$ for all $y \in C$. Such a $P_{C}$ is called the metric projection of $H$ onto $C$. We know that $P_{C}$ is a firmly nonexpansive mapping of $H$ onto $C$, i.e.,

$$
\left\langle x-y, P_{C} x-P_{C} y\right\rangle \geq\left\|P_{C} x-P_{C} y\right\|^{2}, \quad \forall x, y \in H .
$$

Further, for any $x \in H$ and $z \in C, z=P_{C} x$ if and only if

$$
\langle x-z, z-y\rangle \geq 0, \quad \forall y \in C
$$

It is also known that $H$ satisfies Opial's condition [17], that is, if for each sequence $\left\{x_{n}\right\}_{n=1}^{\infty}$ in $H$ which converges weakly to a point $x \in H$, we have

$$
\liminf _{n \rightarrow \infty}\left\|x_{n}-x\right\|<\liminf _{n \rightarrow \infty}\left\|x_{n}-y\right\|, \quad \forall y \in H, y \neq x
$$

In order to prove our main results in the next section, we need the following lemmas.

Lemma 2.1 ([6]) Let $C$ be a nonempty closed convex subset of $H$. Let $\theta: C \times C \rightarrow \mathbb{R}$ be a bifunction satisfying conditions (H1)-(H4) and let $\varphi: C \rightarrow \mathbb{R}$ be a lower semicontinuous and convex function. For $r>0$ and $x \in H$ define a mapping

$$
T_{r}^{(\theta, \varphi)}(x)=\left\{z \in C: \theta(z, y)+\varphi(y)-\varphi(z)+\frac{1}{r}\langle y-z, z-x\rangle \geq 0, \forall y \in C\right\}
$$

for all $x \in H$. Assume that either (A1) or (A2) holds. 
Then the following results hold:

(i) $T_{r}^{(\theta, \varphi)}(x) \neq \phi$ for each $x \in H$ and $T_{r}^{(\theta, \varphi)}$ is single-valued;

(ii) $T_{r}^{(\theta, \varphi)}$ is firmly nonexpansive, i.e., for any $x, y \in H$

$$
\left\|T_{r}^{(\theta, \varphi)} x-T_{r}^{(\theta, \varphi)} y\right\|^{2} \leq\left\langle T_{r}^{(\theta, \varphi)} x-T_{r}^{(\theta, \varphi)} y, x-y\right|
$$

(iii) $F\left(T_{r}^{(\theta, \varphi)}\right)=\operatorname{MEP}(\theta, \varphi)$;

(iv) $\operatorname{MEP}(\theta, \varphi)$ is closed and convex.

By similar argument as in the proof of Lemma 2.2 in [6], we have the following result.

Lemma 2.2 Let $C$ be a nonempty closed convex subset of $H$. Let $G_{1}, G_{2}: C \times C \rightarrow \mathbb{R}$ be two bifunctions satisfying conditions (H1)-(H4). Let $\phi, \psi: C \rightarrow \mathbb{R}$ be two lower semicontinuous and convex functions with restriction (A1) or (A2) and let the mappings $B_{1}, B_{2}: C \rightarrow H$ be $\beta_{1}$-inverse strongly monotone and $\beta_{2}$-inverse strongly monotone, respectively. Let $\mu_{1} \in$ $\left(0,2 \beta_{1}\right)$ and $\mu_{2} \in\left(0,2 \beta_{2}\right)$. Then for given $\bar{x}, \bar{y} \in C,(\bar{x}, \bar{y})$ is a solution of problem (1.5) if and only if $\bar{x}$ is a fixed point of the mapping $\Gamma: C \rightarrow C$ defined by

$$
\Gamma(x)=T_{\mu_{1}}^{\left(G_{1}, \phi\right)}\left[T_{\mu_{2}}^{\left(G_{2}, \psi\right)}\left(x-\mu_{2} B_{2} x\right)-\mu_{1} B_{1} T_{\mu_{2}}^{\left(G_{2}, \psi\right)}\left(x-\mu_{2} B_{2} x\right)\right], \quad \forall x \in C,
$$

where $\bar{y}=T_{\mu_{2}}^{\left(G_{2}, \psi\right)}\left(\bar{x}-\mu_{2} B_{2} \bar{x}\right)$.

Remark 2.1 Under the conditions of Lemma 2.2, the set of fixed points of the mapping $\Gamma$ is denoted by $\Omega$.

Proposition 2.1 ([7]) Let C, H, $\theta, \varphi$ and $T_{r}^{(\theta, \varphi)}$ be as in Lemma 2.1. Then the following holds:

$$
\left\|T_{s}^{(\theta, \varphi)} x-T_{t}^{(\theta, \varphi)} x\right\|^{2} \leq \frac{s-t}{s}\left\langle T_{s}^{(\theta, \varphi)} x-T_{t}^{(\theta, \varphi)} x, T_{s}^{(\theta, \varphi)} x-x\right\rangle
$$

for all $s, t>0$ and $x \in H$.

Lemma 2.3 ([18]) Assume that $T$ is a nonexpansive self-mapping of a nonempty closed convex subset of $C$ of a real Hilbert space $H$. If $T$ has a fixed point, then $I-T$ is demiclosed, that is, when $\left\{x_{n}\right\}_{n=1}^{\infty}$ is a sequence in $C$ converging weakly to some $x \in C$ and the sequence $\left\{(I-T) x_{n}\right\}_{n=1}^{\infty}$ converges strongly to some $y$, it follows that $(I-T) x=y$.

Lemma 2.4 ([4]) Let $M: H \rightarrow 2^{H}$ be a maximal monotone mapping and let $B: H \rightarrow H$ be a monotone and Lipschitz continuous mapping. Then the mapping $M+B: H \rightarrow 2^{H}$ is a maximal monotone mapping.

Lemma 2.5 ([19]) Let $C$ be a nonempty bounded closed convex subset of a Hilbert space $H$ and let $\Im=\{T(s): 0 \leq s<\infty\}$ be a nonexpansive semigroup on $C$. Then for any $h \geq 0$,

$$
\lim _{t \rightarrow \infty} \sup _{x \in C}\left\|\frac{1}{t} \int_{0}^{t} T(s) x d s-T(h)\left(\frac{1}{t} \int_{0}^{t} T(s) x d s\right)\right\|=0 .
$$


Lemma 2.6 ([12]) Let $\left\{a_{n}\right\}_{n=1}^{\infty}$ be a sequence of nonnegative real numbers such that

$$
a_{n+1} \leq\left(1-\delta_{n}\right) a_{n}+b_{n}+\sigma_{n}, \quad n \in N,
$$

where $\left\{\delta_{n}\right\}_{n=1}^{\infty} \subset(0,1)$ and $\left\{b_{n}\right\}_{n=1}^{\infty},\left\{\sigma_{n}\right\}_{n=1}^{\infty}$ are sequences in $\mathbb{R}$ such that

(i) $\lim _{n \rightarrow \infty} \delta_{n}=0, \sum_{n=1}^{\infty} \delta_{n}=\infty$;

(ii) $\lim \sup _{n \rightarrow \infty} \frac{b_{n}}{\delta_{n}} \leq 0$;

(iii) $\sigma_{n} \geq 0, \sum_{n=1}^{\infty} \sigma_{n}<\infty$.

Then $\lim _{n \rightarrow \infty} a_{n}=0$.

Lemma 2.7 ([19]) Assume that $A$ is a strongly positive linear bounded operator on a Hilbert space $H$ with coefficient $\bar{\gamma}>0$ and $0<\rho \leq\|A\|^{-1}$. Then $\|I-\rho A\| \leq 1-\rho \bar{\gamma}$.

\section{Main results}

Now we state and prove our main results.

Theorem 3.1 Let $C$ be a nonempty closed convex subset of a real Hilbert space $H$. Let $\theta, G_{1}, G_{2}: C \times C \rightarrow \mathbb{R}$ be three bifunctions which satisfy assumptions (H1)-(H4) and $\varphi, \phi, \psi: C \rightarrow \mathbb{R}$ be three lower semicontinuous and convex functions with restriction (A1) or (A2). Let $F, B, B_{1}, B_{2}: C \rightarrow H$ be $\zeta$-inverse strongly monotone, $\beta$-inverse strongly monotone, $\beta_{1}$-inverse strongly monotone and $\beta_{2}$-inverse strongly monotone, respectively and $M: H \rightarrow 2^{H}$ be a maximal monotone mapping. Let $\Im=\{T(u): 0 \leq u<\infty\}$ be a oneparameter nonexpansive semigroup on $H$ such that $\mathcal{F}=F(\Im) \cap G M E P \cap \Omega \cap I(B, M) \neq \phi$. Let $\left\{t_{n}\right\} \subset(0, \infty)$ be a real sequence such that $\lim _{n \rightarrow \infty} t_{n}=\infty$. Let $f$ be a contraction from $C$ into itself with a constant $\alpha(0<\alpha<1)$ and let $A$ be a strongly positive linear bounded operator with coefficient $\bar{\gamma}>0$ such that $\|A\| \leq 1$. Assume that $0<\gamma<\frac{\bar{\gamma}}{\alpha\left(1-\alpha_{n} \bar{\gamma}\right)}$. Let $x_{1} \in C$ and let $\left\{x_{n}\right\}$ be a sequence defined by

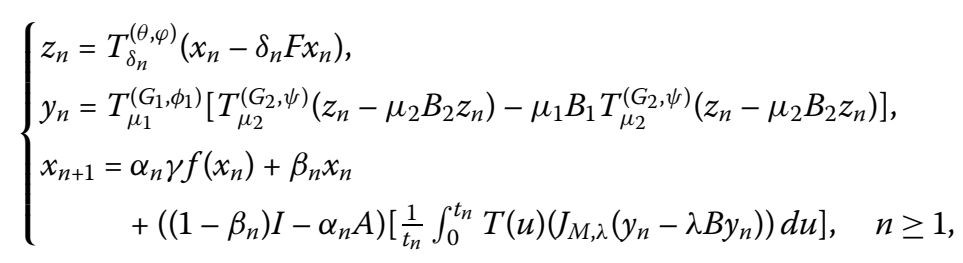

where $\alpha_{n} \in(0,1), \mu_{1} \in\left(0,2 \beta_{1}\right], \mu_{2} \in\left(0,2 \beta_{2}\right], \lambda \in(0,2 \beta], \delta_{n} \in(0,2 \zeta]$ satisfy the following conditions:

(C1) $\lim _{n \rightarrow \infty} \alpha_{n}=0, \sum_{n=1}^{\infty} \alpha_{n}=\infty$ and $\sum_{n=1}^{\infty}\left|\alpha_{n+1}-\alpha_{n}\right|<\infty$;

(C2) $\sum_{n=1}^{\infty} \beta_{n}<\infty$ and $\sum_{n=1}^{\infty}\left|\beta_{n+1}-\beta_{n}\right|<\infty$;

(C3) $\liminf _{n \rightarrow \infty} \delta_{n}>0$ and $\sum_{n=1}^{\infty}\left|\delta_{n+1}-\delta_{n}\right|<\infty$;

(C4) $\lim _{n \rightarrow \infty} \frac{\left|t_{n}-t_{n-1}\right|}{t_{n}} \frac{1}{\alpha_{n}}=0$.

Then $\left\{x_{n}\right\}$ converges strongly to $x^{*}=P_{\mathcal{F}}(\gamma f+(I-A))\left(x^{*}\right)$, which solves the following variational inequality:

$$
\left\langle(A-\gamma f) x^{*}, x-x^{*}\right\rangle \geq 0, \quad \forall x \in \mathcal{F},
$$

and $\left(x^{*}, y^{*}\right)$ is a solution of problem (1.5), where $y^{*}=T_{\mu_{2}}^{\left(G_{2}, \psi\right)}\left(x^{*}-\mu_{2} B_{2} x^{*}\right)$. 
Proof Since $F$ is a $\zeta$-inverse strongly monotone mapping, we have

$$
\begin{aligned}
\left\|\left(I-\delta_{n} F\right) x-\left(I-\delta_{n} F\right) y\right\|^{2} & =\|x-y\|^{2}-2 \delta_{n}\langle x-y, F x-F y\rangle+\delta_{n}^{2}\|F x-F y\|^{2} \\
& \leq\|x-y\|^{2}+\delta_{n}\left(\delta_{n}-2 \zeta\right)\|F x-F y\|^{2} .
\end{aligned}
$$

In similar way, we can obtain

$$
\begin{aligned}
& \|(I-\lambda B) x-(I-\lambda B) y\| \leq\|x-y\|^{2}+\lambda(\lambda-2 \beta)\|B x-B y\|^{2}, \\
& \left\|\left(I-\mu_{1} B_{1}\right) x-\left(I-\mu_{1} B_{1}\right) y\right\| \leq\|x-y\|^{2}+\mu_{1}\left(\mu_{1}-2 \beta_{1}\right)\left\|B_{1} x-B_{1} y\right\|^{2}, \\
& \left\|\left(I-\mu_{2} B_{2}\right) x-\left(I-\mu_{2} B_{2}\right) y\right\| \leq\|x-y\|^{2}+\mu_{2}\left(\mu_{2}-2 \beta_{2}\right)\left\|B_{2} x-B_{2} y\right\|^{2} .
\end{aligned}
$$

Noticing that $\lim _{n \rightarrow \infty} \alpha_{n}=0=\lim _{n \rightarrow \infty} \beta_{n}$, we may assume, with no loss of generality, that $\frac{\alpha_{n}}{1-\beta_{n}}<\|A\|^{-1}$ for all $n \geq 1$. From Lemma 2.7 we know that if $0 \leq \rho \leq\|A\|^{-1}$, then $\|I-\rho A\| \leq$ $1-\rho \bar{\gamma}$. Since $A$ is a strongly positive bounded linear operator on $H$, we have

$$
\|A\|=\sup \{|\langle A x, x\rangle|: x \in H,\|x\|=1\}
$$

Observe that

$$
\left\langle\left(\left(1-\beta_{n}\right) I-\alpha_{n} A\right) x, x\right\rangle=1-\beta_{n}-\alpha_{n}\langle A x, x\rangle \geq 1-\beta_{n}-\alpha_{n}\|A\| \geq 0 .
$$

This shows that $\left(1-\beta_{n}\right) I-\alpha_{n} A$ is positive. It follows that

$$
\begin{aligned}
\left\|\left(1-\beta_{n}\right) I-\alpha_{n} A\right\| & =\sup \left\{\left\langle\left(\left(1-\beta_{n}\right) I-\alpha_{n} A\right) x, x\right\rangle: x \in H,\|x\|=1\right\} \\
& =\sup \left\{1-\beta_{n}-\alpha_{n}\langle A x, x\rangle: x \in H,\|x\|=1\right\} \\
& \leq 1-\beta_{n}-\alpha_{n} \bar{\gamma} .
\end{aligned}
$$

We divide the proof into several steps.

Step 1. $\left\{x_{n}\right\}$ is bounded.

Indeed, take $p \in \mathcal{F}$ arbitrarily. Since $p=T_{\delta_{n}}^{(\theta, \varphi)}\left(p-\delta_{n} F p\right), F$ is $\zeta$-inverse strongly monotone and $0<\delta_{n} \leq 2 \zeta$, we obtain for any $n \geq 1$

$$
\begin{aligned}
\left\|z_{n}-p\right\|^{2} & =\left\|T_{\delta_{n}}^{(\theta, \varphi)}\left(x_{n}-\delta_{n} F x_{n}\right)-T_{\delta_{n}}^{(\theta, \varphi)}\left(p-\delta_{n} F p\right)\right\|^{2} \\
& \leq\left\|\left(x_{n}-p\right)-\delta_{n}\left(F x_{n}-F p\right)\right\|^{2} \\
& =\left\|x_{n}-p\right\|^{p}-2 \delta_{n}\left\langle x_{n}-p, F x_{n}-F p\right\rangle+\delta_{n}^{2}\left\|F x_{n}-F p\right\|^{2} \\
& \leq\left\|x_{n}-p\right\|^{2}+\delta_{n}\left(\delta_{n}-2 \zeta\right)\left\|F x_{n}-F p\right\|^{2} \\
& \leq\left\|x_{n}-p\right\|^{2} .
\end{aligned}
$$

Putting $u_{n}=T_{\mu_{2}}^{\left(G_{2}, \psi\right)}\left(z_{n}-\mu_{2} B_{2} z_{n}\right), w_{n}=J_{M, \lambda}\left(y_{n}-\lambda B y_{n}\right)$, and $u=T_{\mu_{2}}^{\left(G_{2}, \psi\right)}\left(p-\mu_{2} B_{2} p\right)$, we have

$$
\begin{aligned}
\left\|u_{n}-u\right\|^{2} & =\left\|T_{\mu_{2}}^{\left(G_{2}, \psi\right)}\left(z_{n}-\mu_{2} B_{2} z_{n}\right)-T_{\mu_{2}}^{\left(G_{2}, \mu\right)}\left(p-\mu_{2} B_{2} p\right)\right\|^{2} \\
& \leq\left\|\left(z_{n}-p\right)-\mu_{2}\left(B_{2} z_{n}-B_{2} p\right)\right\|^{2}
\end{aligned}
$$




$$
\begin{aligned}
& =\left\|z_{n}-p\right\|^{2}-2 \mu_{2}\left\langle z_{n}-p, B_{2} z_{n}-B_{2} p\right\rangle+\mu_{2}^{2}\left\|B_{2} z_{n}-B_{2} p\right\|^{2} \\
& \leq\left\|z_{n}-p\right\|^{2}+\mu_{2}\left(\mu_{2}-2 \beta_{2}\right)\left\|B_{2} z_{n}-B_{2} p\right\|^{2} \\
& \leq\left\|z_{n}-p\right\|^{2}
\end{aligned}
$$

and

$$
\begin{aligned}
\left\|w_{n}-p\right\|^{2} & =\left\|J_{M, \lambda}\left(y_{n}-\lambda B y_{n}\right)-J_{M, \lambda}(p-\lambda B p)\right\|^{2} \\
& \leq\left\|\left(y_{n}-p\right)-\lambda\left(B y_{n}-B p\right)\right\|^{2} \\
& =\left\|y_{n}-p\right\|^{2}-2 \lambda\left\langle y_{n}-p, B y_{n}-B p\right\rangle+\lambda^{2}\left\|B y_{n}-B p\right\|^{2} \\
& \leq\left\|y_{n}-p\right\|^{2}+\lambda(\lambda-2 \beta)\left\|B y_{n}-B p\right\|^{2} \\
& \leq\left\|y_{n}-p\right\|^{2} .
\end{aligned}
$$

And since

$$
\begin{aligned}
p & =T_{\mu_{1}}^{\left(G_{1}, \phi\right)}\left[T_{\mu_{2}}^{\left(G_{2}, \psi\right)}\left(p-\mu_{2} B_{2} p\right)-\mu_{1} B_{1} T_{\mu_{2}}^{\left(G_{2}, \psi\right)}\left(p-\mu_{2} B_{2} p\right)\right] \\
& =T_{\mu_{1}}^{\left(G_{1}, \phi\right)}\left(u-\mu_{1} B_{1} u\right),
\end{aligned}
$$

we know that for any $n \geq 1$

$$
\begin{aligned}
\left\|y_{n}-p\right\|^{2} & =\left\|T_{\mu_{1}}^{\left(G_{1}, \phi\right)}\left(u_{n}-\mu_{1} B_{1} u_{n}\right)-T_{\mu_{1}}^{\left(G_{1}, \phi\right)}\left(u-\mu_{1} B_{1} u\right)\right\|^{2} \\
& \leq\left\|\left(u_{n}-u\right)-\mu_{1}\left(B_{1} u_{n}-B_{1} u\right)\right\|^{2} \\
& =\left\|u_{n}-u\right\|^{2}-2 \mu_{1}\left\langle u_{n}-u, B_{1} u_{n}-B_{1} u\right\rangle+\mu_{1}^{2}\left\|B_{1} u_{n}-B_{1} u\right\|^{2} \\
& \leq\left\|u_{n}-u\right\|^{2}+\mu_{1}\left(\mu_{1}-2 \beta_{1}\right)\left\|B_{1} u_{n}-B_{1} u\right\|^{2} \\
& \leq\left\|u_{n}-u\right\|^{2} \\
& \leq\left\|z_{n}-p\right\|^{2} .
\end{aligned}
$$

Since $A$ is a strongly positive linear bounded operator with coefficient $\bar{\gamma}>0$, we have

$$
\begin{aligned}
& \left\|x_{n+1}-p\right\| \\
& \quad=\left\|\alpha_{n}\left(\gamma f\left(x_{n}\right)-A p\right)+\beta_{n}\left(x_{n}-p\right)+\left(\left(1-\beta_{n}\right) I-\alpha_{n} A\right) \frac{1}{t_{n}} \int_{0}^{t_{n}}\left[T(u) w_{n}-T(u) p\right] d u\right\| \\
& \quad \leq \alpha_{n}\left\|\gamma f\left(x_{n}\right)-A p\right\|+\beta_{n}\left\|x_{n}-p\right\|+\left(1-\beta_{n}-\alpha_{n} \bar{\gamma}\right)\left\|w_{n}-p\right\| \\
& \quad \leq \alpha_{n} \gamma\left\|f\left(x_{n}\right)-f(p)\right\|+\alpha_{n}\|\gamma f(p)-A p\|+\beta_{n}\left\|x_{n}-p\right\|+\left(1-\beta_{n}-\alpha_{n} \bar{\gamma}\right)\left\|x_{n}-p\right\| \\
& \quad \leq\left[1-\alpha_{n}(\bar{\gamma}-\gamma \alpha)\right]\left\|x_{n}-p\right\|+\alpha_{n}\|\gamma f(p)-A p\| .
\end{aligned}
$$

By induction, we obtain for all $n \geq 1$

$$
\left\|x_{n}-p\right\| \leq \max \left\{\left\|x_{1}-p\right\|, \frac{1}{\bar{\gamma}-\gamma \alpha}\|\gamma f(p)-A p\|\right\} .
$$

Hence $\left\{x_{n}\right\}_{n=1}^{\infty}$ is bounded. So are $\left\{y_{n}\right\}_{n=1}^{\infty},\left\{z_{n}\right\}_{n=1}^{\infty},\left\{w_{n}\right\}_{n=1}^{\infty}$. 
Step 2. We show that $\lim _{n \rightarrow \infty}\left\|x_{n+1}-x_{n}\right\|=0$.

We estimate $\left\|y_{n+1}-y_{n}\right\|$. From (3.1), we have

$$
\begin{aligned}
&\left\|y_{n+1}-y_{n}\right\|^{2}=\left\|T_{\mu_{1}}^{\left(G_{1}, \phi\right)}\left(u_{n+1}-\mu_{1} B_{1} u_{n+1}\right)-T_{\mu_{1}}^{\left(G_{1}, \phi\right)}\left(u_{n}-\mu_{1} B_{1} u_{n}\right)\right\|^{2} \\
& \leq\left\|\left(u_{n+1}-u_{n}\right)-\mu_{1}\left(B_{1} u_{n+1}-B_{1} u_{n}\right)\right\|^{2} \\
& \leq\left\|u_{n+1}-u_{n}\right\|^{2}+\mu_{1}\left(\mu_{1}-2 \beta_{1}\right)\left\|B_{1} u_{n+1}-B_{1} u_{n}\right\|^{2} \\
& \leq\left\|u_{n+1}-u_{n}\right\|^{2} \\
&=\left\|T_{\mu_{2}}^{\left(G_{2}, \psi\right)}\left(z_{n+1}-\mu_{2} B_{2} z_{n+1}\right)-T_{\mu_{2}}^{\left(G_{2}, \psi\right)}\left(z_{n}-\mu_{2} B_{2} z_{n}\right)\right\|^{2} \\
& \leq\left\|\left(z_{n+1}-z_{n}\right)-\mu_{2}\left(B_{2} z_{n+1}-B_{2} z_{n}\right)\right\|^{2} \\
& \leq\left\|z_{n+1}-z_{n}\right\|^{2}+\mu_{2}\left(\mu_{2}-2 \beta_{2}\right)\left\|B_{2} z_{n+1}-B_{2} z_{n}\right\|^{2} \\
& \leq\left\|z_{n+1}-z_{n}\right\|^{2}, \\
&\left\|\left(x_{n+1}-\delta_{n+1} F x_{n+1}\right)-\left(x_{n}-\delta_{n} F x_{n}\right)\right\| \\
&=\left\|x_{n+1}-x_{n}-\delta_{n+1}\left(F x_{n+1}-F x_{n}\right)+\left(\delta_{n}-\delta_{n+1}\right) F x_{n}\right\| \\
& \leq\left\|x_{n+1}-x_{n}-\delta_{n+1}\left(F x_{n+1}-F x_{n}\right)\right\|+\left|\delta_{n}-\delta_{n+1}\right|\left\|F x_{n}\right\| \\
& \leq\left\|x_{n+1}-x_{n}\right\|+\left|\delta_{n}-\delta_{n+1}\right|\left\|F x_{n}\right\|
\end{aligned}
$$

and

$$
\begin{aligned}
\left\|z_{n+1}-z_{n}\right\|= & \left\|T_{\delta_{n+1}}^{(\theta, \varphi)}\left(x_{n+1}-\delta_{n+1} F x_{n+1}\right)-T_{\delta_{n}}^{(\theta, \varphi)}\left(x_{n}-\delta_{n} F x_{n}\right)\right\| \\
\leq & \left\|T_{\delta_{n+1}}^{(\theta, \varphi)}\left(x_{n+1}-\delta_{n+1} F x_{n+1}\right)-T_{\delta_{n+1}}^{(\theta, \varphi)}\left(x_{n}-\delta_{n} F x_{n}\right)\right\| \\
& +\left\|T_{\delta_{n+1}}^{(\theta, \varphi)}\left(x_{n}-\delta_{n} F x_{n}\right)-T_{\delta_{n}}^{(\theta, \varphi)}\left(x_{n}-\delta_{n} F x_{n}\right)\right\| \\
\leq & \left\|\left(x_{n+1}-\delta_{n+1} F x_{n+1}\right)-\left(x_{n}-\delta_{n} F x_{n}\right)\right\| \\
& +\left\|T_{\delta_{n+1}}^{(\theta, \varphi)}\left(x_{n}-\delta_{n} F x_{n}\right)-T_{\delta_{n}}^{(\theta, \varphi)}\left(x_{n}-\delta_{n} F x_{n}\right)\right\| \\
\leq & \left\|x_{n+1}-x_{n}\right\|+\left|\delta_{n+1}-\delta_{n}\right|\left\|F x_{n}\right\| \\
& +\left\|T_{\delta_{n+1}}^{(\theta, \varphi)}\left(x_{n}-\delta_{n} F x_{n}\right)-T_{\delta_{n}}^{(\theta, \varphi)}\left(x_{n}-\delta_{n} F x_{n}\right)\right\| .
\end{aligned}
$$

Without loss of generality, let us assume that there exists a real number $a$ such that $\delta_{n}>$ $a>0$ for all $n$. Utilizing Proposition 2.1 we have

$$
\begin{array}{r}
\left\|T_{\delta_{n+1}}^{(\theta, \varphi)}\left(x_{n}-\delta_{n} F x_{n}\right)-T_{\delta_{n}}^{(\theta, \varphi)}\left(x_{n}-\delta_{n} F x_{n}\right)\right\| \\
\leq \frac{\left|\delta_{n+1}-\delta_{n}\right|}{\delta_{n+1}}\left\|T_{\delta_{n+1}}^{(\theta, \varphi)}\left(I-\delta_{n} F\right) x_{n}-x_{n}\right\| \\
\leq \frac{\left|\delta_{n+1}-\delta_{n}\right|}{a}\left\|T_{\delta_{n+1}}^{(\theta, \varphi)}\left(I-\delta_{n} F\right) x_{n}-x_{n}\right\| .
\end{array}
$$

It follows from (3.8)-(3.10) that

$$
\begin{aligned}
\left\|w_{n+1}-w_{n}\right\| & =\left\|J_{M, \lambda}\left(y_{n+1}-\lambda B u_{n+1}\right)-J_{M, \lambda}\left(y_{n}-\lambda B y_{n}\right)\right\| \\
& \leq\left\|\left(y_{n+1}-\lambda B y_{n+1}\right)-\left(y_{n}-\lambda B y_{n}\right)\right\| \\
& \leq\left\|y_{n+1}-y_{n}\right\|
\end{aligned}
$$


and

$$
\begin{aligned}
\left\|y_{n+1}-y_{n}\right\| \leq & \left\|z_{n+1}-z_{n}\right\| \\
\leq & \left\|x_{n+1}-x_{n}\right\|+\left|\delta_{n+1}-\delta_{n}\right|\left\|F x_{n}\right\| \\
& \quad+\frac{\left|\delta_{n+1}-\delta_{n}\right|}{a}\left\|T_{\delta_{n+1}}^{(\theta, \varphi)}\left(I-\delta_{n} F\right) x_{n}-x_{n}\right\| .
\end{aligned}
$$

Put $h_{n}=\frac{1}{t_{n}} \int_{0}^{t_{n}} T(u) w_{n} d u$ for all $n \geq 1$. We note that

$$
\begin{aligned}
\left\|h_{n}-h_{n-1}\right\|= & \left\|\frac{1}{t_{n}} \int_{0}^{t_{n}} T(u) w_{n} d u-\frac{1}{t_{n-1}} \int_{0}^{t_{n-1}} T(u) w_{n-1} d u\right\| \\
\leq & \left\|\frac{1}{t_{n}} \int_{0}^{t_{n}}\left[T(u) w_{n}-T(u) w_{n-1}\right] d u\right\| \\
& +\left|\frac{1}{t_{n}}-\frac{1}{t_{n-1}}\right|\left\|\int_{0}^{t_{n-1}}\left[T(u) w_{n-1}-T(u) p\right] d u\right\| \\
& +\frac{1}{t_{n}}\left\|\int_{t_{n-1}}^{t_{n}}\left[T(u) w_{n-1}-T(u) p\right] d u\right\| \\
\leq & \left\|y_{n}-y_{n-1}\right\|+\frac{2\left|t_{n}-t_{n-1}\right|}{t_{n}}\left\|y_{n-1}-p\right\| .
\end{aligned}
$$

Using (3.12) and (3.13) we get

$$
\begin{aligned}
\| x_{n+1} & -x_{n} \| \\
= & \|\left[\alpha_{n} \gamma f\left(x_{n}\right)+\beta_{n} x_{n}+\left(\left(1-\beta_{n}\right) I-\alpha_{n} A\right) h_{n}\right] \\
& -\left[\alpha_{n-1} \gamma f\left(x_{n-1}\right)+\beta_{n-1} x_{n-1}+\left(\left(1-\beta_{n-1}\right) I-\alpha_{n-1} A\right) h_{n-1}\right] \| \\
\leq & \alpha_{n} \gamma \alpha\left\|x_{n}-x_{n-1}\right\|+\gamma\left|\alpha_{n}-\alpha_{n-1}\right|\left\|f\left(x_{n-1}\right)\right\|+\beta_{n}\left\|x_{n}-x_{n-1}\right\| \\
& +\left|\beta_{n}-\beta_{n-1}\right|\left\|x_{n-1}\right\|+\left(1-\alpha_{n} \bar{\gamma}\right)\left\|h_{n}-h_{n-1}\right\|+\left|\alpha_{n}-\alpha_{n-1}\right|\left\|A h_{n-1}\right\| \\
& +\left|\beta_{n}-\beta_{n-1}\right|\left\|h_{n}-h_{n-1}\right\| \\
\leq & \alpha_{n} \gamma \alpha\left\|x_{n}-x_{n-1}\right\|+\gamma\left|\alpha_{n}-\alpha_{n-1}\right|\left\|f\left(x_{n-1}\right)\right\|+\beta_{n}\left\|x_{n}-x_{n-1}\right\| \\
& +\left|\beta_{n}-\beta_{n-1}\right|\left\|x_{n-1}\right\|+\left(1-\alpha_{n} \bar{\gamma}\right)\left[\left\|y_{n}-y_{n-1}\right\|+\frac{2\left|t_{n}-t_{n-1}\right|}{t_{n}}\left\|y_{n-1}-p\right\|\right] \\
& +\left|\alpha_{n}-\alpha_{n-1}\right|\left\|A h_{n-1}\right\|+\left|\beta_{n}-\beta_{n-1}\right|\left[\left\|y_{n}-y_{n-1}\right\|+\frac{2\left|t_{n}-t_{n-1}\right|}{t_{n}}\left\|y_{n-1}-p\right\|\right] \\
\leq & \alpha_{n} \gamma \alpha\left\|x_{n}-x_{n-1}\right\|+\gamma\left|\alpha_{n}-\alpha_{n-1}\right|\left\|f\left(x_{n-1}\right)\right\|+\beta_{n}\left\|x_{n}-x_{n-1}\right\| \\
& +\left|\beta_{n}-\beta_{n-1}\right|\left[\left\|x_{n}-x_{n-1}\right\|+\left|\delta_{n}-\delta_{n-1}\right|\left\|F x_{n-1}\right\|\right. \\
& +\left|\beta_{n}-\beta_{n-1}\right|\left\|x_{n-1}\right\|+\left(1-\alpha_{n} \bar{\gamma}\right)\left[\left\|x_{n}-x_{n-1}\right\|+\left|\delta_{n}-\delta_{n-1}\right|\left\|F x_{n-1}\right\|\right. \\
& \left.+\frac{\left|\delta_{n}-\delta_{n-1}\right|}{a}\left\|T_{\delta_{n}}^{(\theta, \varphi)}\left(I-\delta_{n-1} F\right) x_{n-1}-x_{n-1}\right\|\right] \\
& +\left(1-\alpha_{n} \bar{\gamma}\right) \frac{2\left|t_{n}-t_{n-1}\right|\left\|y_{n-1}-p\right\|+\left|\alpha_{n}-\alpha_{n-1}\right|\left\|A h_{n-1}\right\|}{t_{n}}
\end{aligned}
$$




$$
\begin{aligned}
& \left.+\frac{\left|\delta_{n}-\delta_{n-1}\right|}{a}\left\|T_{\delta_{n}}^{(\theta, \varphi)}\left(I-\delta_{n-1} F\right) x_{n-1}-x_{n-1}\right\|\right] \\
& +\left|\beta_{n}-\beta_{n-1}\right| \frac{2\left|t_{n}-t_{n-1}\right|}{t_{n}}\left\|y_{n-1}-p\right\| \\
\leq & {\left[1-(\bar{\gamma}-\gamma \alpha) \alpha_{n}\right]\left\|x_{n}-x_{n-1}\right\|+\frac{4\left|t_{n}-t_{n-1}\right|}{t_{n}}\left\|y_{n-1}-p\right\| } \\
& +\left|\alpha_{n}-\alpha_{n-1}\right|\left(\gamma\left\|f\left(x_{n-1}\right)\right\|+\left\|A h_{n-1}\right\|\right)+\beta_{n}\left[\left\|x_{n}\right\|+\left\|x_{n-1}\right\|\right] \\
& +\left|\beta_{n}-\beta_{n-1}\right|\left[\left\|x_{n}\right\|+2\left\|x_{n-1}\right\|\right] \\
& +2\left|\delta_{n}-\delta_{n-1}\right|\left[\left\|F x_{n-1}\right\|+\frac{1}{a}\left\|T_{\delta_{n}}^{(\theta, \varphi)}\left(I-\delta_{n-1} F\right) x_{n-1}-x_{n-1}\right\|\right] \\
= & {\left[1-(\bar{\gamma}-\gamma \alpha) \alpha_{n}\right]\left\|x_{n}-x_{n-1}\right\|+D\left[\frac{4\left|t_{n}-t_{n-1}\right|}{t_{n}}+\left|\alpha_{n}-\alpha_{n-1}\right|+\beta_{n}\right.} \\
& \left.+\left|\beta_{n}-\beta_{n-1}\right|+2\left|\delta_{n}-\delta_{n-1}\right|\right]
\end{aligned}
$$

where $D=\max \left\{3 \sup _{n \geq 1}\left\|x_{n}\right\|, \sup _{n \geq 1}\left(\gamma\left\|f\left(x_{n}\right)\right\|+\left\|A h_{n}\right\|\right), \sup _{n \geq 1}\left(\left\|F x_{n}\right\|+\frac{1}{a} \| T_{\delta_{n}}^{(\theta, \varphi)}\left(I-\delta_{n} F\right) \times\right.\right.$ $\left.x_{n}-x_{n} \|\right)$, $\left.\sup _{n \geq 1}\left\|y_{n}-p\right\|\right\}$. From Lemma 2.6, taking $\delta_{n}=(\bar{\gamma}-\gamma \alpha) \alpha_{n}, b_{n}=\frac{4\left|t_{n}-t_{n-1}\right|}{t_{n}} D, \sigma_{n}=$ $D\left(\left|\alpha_{n}-\alpha_{n-1}\right|+\beta_{n}+\left|\beta_{n}-\beta_{n-1}\right|+2\left|\delta_{n}-\delta_{n-1}\right|\right)$, it follows that $\lim _{n \rightarrow \infty}\left\|x_{n+1}-x_{n}\right\|=0$.

Step 3. $\lim _{n \rightarrow \infty}\left\|F x_{n}-F p\right\|=0, \lim _{n \rightarrow \infty}\left\|B_{1} u_{n}-B_{1} u\right\|=0, \lim _{n \rightarrow \infty}\left\|B_{2} z_{n}-B_{2} p\right\|=0$ and $\lim _{n \rightarrow \infty}\left\|B y_{n}-B p\right\|=0$.

Indeed, from (3.1), (3.4), (3.5), and (3.7) we get

$$
\begin{aligned}
\left\|x_{n+1}-p\right\|^{2} & \| \alpha_{n}\left(\gamma f\left(x_{n}\right)-A p\right)+\beta_{n}\left(x_{n}-p\right)+\left(\left(1-\beta_{n}\right) I\right. \\
& \left.-\alpha_{n} A\right) \frac{1}{t_{n}} \int_{0}^{t_{n}}\left[T(u)\left(J_{M, \lambda}\left(y_{n}-\lambda B y_{n}\right)\right)-T(u)\left(J_{M, \lambda}(p-\lambda B p)\right)\right] d u \|^{2} \\
\leq & \alpha_{n}\left\|\gamma f\left(x_{n}\right)-A p\right\|^{2}+\beta_{n}\left\|x_{n}-p\right\|^{2}+\left(1-\beta_{n}-\alpha_{n} \bar{\gamma}\right)\left\|y_{n}-p\right\|^{2} \\
\leq & \alpha_{n}\left\|\gamma f\left(x_{n}\right)-A p\right\|^{2}+\beta_{n}\left\|x_{n}-p\right\|^{2} \\
& +\left(1-\beta_{n}-\alpha_{n} \bar{\gamma}\right)\left[\left\|u_{n}-u\right\|^{2}+\mu_{1}\left(\mu_{1}-2 \beta_{1}\right)\left\|B_{1} u_{n}-B_{1} u\right\|^{2}\right] \\
\leq & \alpha_{n}\left\|\gamma f\left(x_{n}\right)-A p\right\|^{2}+\beta_{n}\left\|x_{n}-p\right\|^{2}+\left(1-\beta_{n}-\alpha_{n} \bar{\gamma}\right)\left[\left\|z_{n}-p\right\|^{2}\right. \\
& \left.+\mu_{2}\left(\mu_{2}-2 \beta_{2}\right)\left\|B_{2} z_{n}-B_{2} p\right\|^{2}+\mu_{1}\left(\mu_{1}-2 \beta_{1}\right)\left\|B_{1} u_{n}-B_{1} u\right\|^{2}\right] \\
\leq & \alpha_{n}\left\|\gamma f\left(x_{n}\right)-A p\right\|^{2}+\beta_{n}\left\|x_{n}-p\right\|^{2}+\left(1-\beta_{n}-\alpha_{n} \bar{\gamma}\right)\left[\left\|x_{n}-p\right\|^{2}\right. \\
& +\delta_{n}\left(\delta_{n}-2 \zeta\right)\left\|F x_{n}-F p\right\|^{2}+\mu_{2}\left(\mu_{2}-2 \beta_{2}\right)\left\|B_{2} z_{n}-B_{2} p\right\|^{2} \\
& \left.+\mu_{1}\left(\mu_{1}-2 \beta_{1}\right)\left\|B_{1} u_{n}-B_{1} u\right\|^{2}\right] .
\end{aligned}
$$

Therefore

$$
\begin{aligned}
\delta_{n}(2 \zeta & \left.-\delta_{n}\right)\left\|F x_{n}-F p\right\|^{2}+\mu_{2}\left(2 \beta_{2}-\mu_{2}\right)\left\|B_{2} z_{n}-B_{2} p\right\|^{2} \\
& +\mu_{1}\left(2 \beta_{1}-\mu_{1}\right)\left\|B_{1} u_{n}-B_{1} u\right\|^{2}
\end{aligned}
$$




$$
\begin{aligned}
& \leq \alpha_{n}\left\|\gamma f\left(x_{n}\right)-A p\right\|^{2}+\left\|x_{n}-p\right\|^{2}-\left\|x_{n+1}-p\right\|^{2} \\
& \leq \alpha_{n}\left\|\gamma f\left(x_{n}\right)-A p\right\|^{2}+\left(\left\|x_{n}-p\right\|+\left\|x_{n+1}-p\right\|\right)\left\|x_{n}-x_{n+1}\right\| .
\end{aligned}
$$

Since $\alpha_{n} \rightarrow 0$ and $\left\|x_{n}-x_{n+1}\right\| \rightarrow 0$ as $n \rightarrow \infty$, we have $\lim _{n \rightarrow \infty}\left\|F x_{n}-F p\right\|=0$, $\lim _{n \rightarrow \infty}\left\|B_{2} z_{n}-B_{2} p\right\|=0$ and $\lim _{n \rightarrow \infty}\left\|B_{1} u_{n}-B_{1} u\right\|=0$. Similarly, from (3.4), (3.6), and (3.7) we have

$$
\begin{aligned}
\left\|x_{n+1}-p\right\|^{2} & \leq \alpha_{n}\left\|\gamma f\left(x_{n}\right)-A p\right\|^{2}+\beta_{n}\left\|x_{n}-p\right\|^{2}+\left(1-\beta_{n}-\alpha_{n} \bar{\gamma}\right)\left\|w_{n}-p\right\|^{2} \\
& \leq \alpha_{n}\left\|\gamma f\left(x_{n}\right)-A p\right\|^{2}+\left(1-\alpha_{n} \bar{\gamma}\right)\left\|x_{n}-p\right\|^{2}+\lambda(\lambda-2 \beta)\left\|B y_{n}-B p\right\|^{2},
\end{aligned}
$$

which implies that

$$
\lambda(2 \beta-\lambda)\left\|B y_{n}-B p\right\|^{2} \leq \alpha_{n}\left\|\gamma f\left(x_{n}\right)-A p\right\|^{2}+\left(\left\|x_{n}-p\right\|+\left\|x_{n+1}-p\right\|\right)\left\|x_{n}-x_{n+1}\right\| .
$$

We also have $\lim _{n \rightarrow \infty}\left\|B y_{n}-B p\right\|=0$.

Step 4. We claim that $\lim _{n \rightarrow \infty}\left\|x_{n}-y_{n}\right\|=0, \lim _{n \rightarrow \infty}\left\|x_{n}-z_{n}\right\|=0$ and $\lim _{n \rightarrow \infty} \| w_{n}-$ $y_{n} \|=0$.

Indeed, from Lemma 2.1, (3.4), (3.5), and (3.7) we have

$$
\begin{aligned}
\left\|u_{n}-u\right\|^{2}= & \left\|T_{\mu_{2}}^{\left(G_{2}, \psi\right)}\left(z_{n}-\mu_{2} B_{2} z_{n}\right)-T_{\mu_{2}}^{\left(G_{2}, \psi\right)}\left(p-\mu_{2} B_{2} p\right)\right\|^{2} \\
\leq & \left\langle\left(z_{n}-\mu_{2} B_{2} z_{n}\right)-\left(p-\mu_{2} B_{2} p\right), u_{n}-u\right\rangle \\
= & \frac{1}{2}\left[\left\|\left(z_{n}-\mu_{2} B_{2} z_{n}\right)-\left(p-\mu_{2} B_{2} p\right)\right\|^{2}+\left\|u_{n}-u\right\|^{2}\right. \\
& \left.-\left\|\left(z_{n}-\mu_{2} B_{2} z_{n}\right)-\left(p-\mu_{2} B_{2} z_{n}\right)-\left(u_{n}-u\right)\right\|^{2}\right] \\
\leq & \frac{1}{2}\left[\left\|x_{n}-p\right\|^{2}+\left\|u_{n}-u\right\|^{2}-\left\|\left(z_{n}-u_{n}\right)-(p-u)\right\|^{2}\right. \\
& \left.+2 \mu_{2}\left\langle\left(z_{n}-u_{n}\right)-(p-u), B_{2} z_{n}-B_{2} p\right\rangle-\mu_{2}^{2}\left\|B_{2} z_{n}-B_{2} p\right\|^{2}\right]
\end{aligned}
$$

and

$$
\begin{aligned}
\left\|y_{n}-p\right\|^{2}= & \left\|T_{\mu_{1}}^{\left(G_{1}, \phi\right)}\left(u_{n}-\mu_{1} B_{1} u_{n}\right)-T_{\mu_{1}}^{\left(G_{1}, \phi\right)}\left(u-\mu_{1} B_{1} u\right)\right\| \\
\leq & \left\langle\left(u_{n}-\mu B_{1} u_{n}\right)-\left(u-\mu_{1} B_{1} u\right), y_{n}-p\right\rangle \\
= & \frac{1}{2}\left[\left\|\left(u_{n}-\mu_{1} B_{1} u_{n}\right)-\left(u-\mu_{1} B_{1} u\right)\right\|^{2}+\left\|y_{n}-p\right\|^{2}\right. \\
& \left.-\left\|\left(u_{n}-\mu_{1} B_{1} u_{n}\right)-\left(u-\mu_{1} B_{1} u\right)-\left(y_{n}-p\right)\right\|^{2}\right] \\
\leq & \frac{1}{2}\left[\left\|u_{n}-u\right\|^{2}+\left\|y_{n}-p\right\|^{2}-\left\|\left(u_{n}-y_{n}\right)+(p-u)\right\|^{2}\right. \\
& \left.+2 \mu_{1}\left\langle B_{1} u_{n}-B_{1} u,\left(u_{n}-y_{n}\right)+(p-u)\right\rangle-\mu_{1}^{2}\left\|B_{1} u_{n}-B_{1} u\right\|^{2}\right] \\
\leq & \frac{1}{2}\left[\left\|x_{n}-p\right\|^{2}+\left\|y_{n}-p\right\|^{2}-\left\|\left(u_{n}-y_{n}\right)+(p-u)\right\|^{2}\right. \\
& \left.+2 \mu_{1}\left\langle B_{1} u_{n}-B_{1} u,\left(u_{n}-y_{n}\right)+(p-u)\right\rangle\right],
\end{aligned}
$$


which imply that

$$
\begin{aligned}
\left\|u_{n}-u\right\|^{2} \leq & \left\|x_{n}-p\right\|^{2}-\left\|\left(z_{n}-u_{n}\right)-(p-u)\right\|^{2} \\
& +2 \mu_{2}\left\{\left(z_{n}-u_{n}\right)-(p-u), B_{2} z_{n}-B_{2} p\right\rangle-\mu_{1}^{2}\left\|B_{2} z_{n}-B_{2} p\right\|^{2}
\end{aligned}
$$

and

$$
\begin{aligned}
\left\|y_{n}-p\right\|^{2} \leq & \left\|x_{n}-p\right\|^{2}-\left\|\left(u_{n}-y_{n}\right)+(p-u)\right\|^{2} \\
& +2 \mu_{1}\left\|B_{1} u_{n}-B_{1} u\right\|\left\|\left(u_{n}-y_{n}\right)+(p-u)\right\| .
\end{aligned}
$$

It follows from (3.15) that

$$
\begin{aligned}
\left\|x_{n+1}-p\right\|^{2} \leq & \alpha_{n}\left\|\gamma f\left(x_{n}\right)-A p\right\|^{2}+\beta_{n}\left\|x_{n}-p\right\|^{2}+\left(1-\beta_{n}-\alpha_{n} \bar{\gamma}\right)\left\|y_{n}-p\right\|^{2} \\
\leq & \alpha_{n}\left\|\gamma f\left(x_{n}\right)-A p\right\|^{2}+\left(1-\alpha_{n} \bar{\gamma}\right)\left\|x_{n}-p\right\|^{2} \\
& -\left(1-\beta_{n}-\alpha_{n} \bar{\gamma}\right)\left\|\left(u_{n}-y_{n}\right)+(p-u)\right\|^{2} \\
& +2 \mu_{1}\left(1-\beta_{n}-\alpha_{n} \bar{\gamma}\right)\left\|B_{1} u_{n}-B_{1} u\right\|\left\|\left(u_{n}-y_{n}\right)+(p-u)\right\|,
\end{aligned}
$$

which gives

$$
\begin{aligned}
\left(1-\beta_{n}-\alpha_{n} \bar{\gamma}\right)\left\|\left(u_{n}-y_{n}\right)+(p-u)\right\|^{2} & \\
\leq & \alpha_{n}\left\|\gamma f\left(x_{n}\right)-A p\right\|^{2}+\left(\left\|x_{n}-p\right\|+\left\|x_{n+1}-p\right\|\right)\left\|x_{n}-x_{n+1}\right\| \\
& +2 \mu_{1}\left(1-\beta_{n}-\alpha_{n} \bar{\gamma}\right)\left\|B_{1} u_{n}-B_{1} u\right\|\left\|\left(u_{n}-y_{n}\right)+(p-u)\right\| .
\end{aligned}
$$

Since $\alpha_{n} \rightarrow 0,\left\|x_{n+1}-x_{n}\right\| \rightarrow 0$ and $\left\|B_{1} u_{n}-B_{1} u\right\| \rightarrow 0$ as $n \rightarrow \infty$, we have

$$
\lim _{n \rightarrow \infty}\left\|\left(u_{n}-y_{n}\right)+(p-u)\right\|=0 .
$$

Also, from (3.14) we have

$$
\begin{aligned}
\left\|x_{n+1}-p\right\|^{2} \leq & \alpha_{n}\left\|\gamma f\left(x_{n}\right)-A p\right\|^{2}+\beta_{n}\left\|x_{n}-p\right\|^{2}+\left(1-\beta_{n}-\alpha_{n} \bar{\gamma}\right)\left\|y_{n}-p\right\|^{2} \\
\leq & \alpha_{n}\left\|\gamma f\left(x_{n}\right)-A p\right\|^{2}+\beta_{n}\left\|x_{n}-p\right\|^{2}+\left(1-\beta_{n}-\alpha_{n} \bar{\gamma}\right)\left\|u_{n}-u\right\|^{2} \\
\leq & \alpha_{n}\left\|\gamma f\left(x_{n}\right)-A p\right\|^{2}+\beta_{n}\left\|x_{n}-p\right\|^{2}+\left(1-\beta_{n}-\alpha_{n} \bar{\gamma}\right)\left[\left\|x_{n}-p\right\|^{2}\right. \\
& \left.\quad-\left\|\left(z_{n}-u_{n}\right)-(p-u)\right\|^{2}+2 \mu_{2}\left(\left(z_{n}-u_{n}\right)-(p-u), B_{2} z_{n}-B_{2} p\right\rangle\right] .
\end{aligned}
$$

So, we have

$$
\begin{aligned}
(1- & \left.\beta_{n}-\alpha_{n} \bar{\gamma}\right)\left\|\left(z_{n}-u_{n}\right)-(p-u)\right\|^{2} \\
\leq & \alpha_{n}\left\|\gamma f\left(x_{n}\right)-A p\right\|^{2}+\left(\left\|x_{n}-p\right\|+\left\|x_{n+1}-p\right\|\right)\left\|x_{n}-x_{n+1}\right\| \\
& +2 \mu_{2}\left\|\left(z_{n}-u_{n}\right)-(p-u)\right\|\left\|B_{2} z_{n}-B_{2} p\right\| .
\end{aligned}
$$


Note that $\left\|B_{2} z_{n}-B_{2} p\right\| \rightarrow 0$ as $n \rightarrow \infty$. Then we have

$$
\lim _{n \rightarrow \infty}\left\|\left(z_{n}-u_{n}\right)-(p-u)\right\|=0
$$

In addition, from the firm nonexpansivity of $T_{\delta_{n}}^{(\theta, \varphi)}$, we obtain

$$
\begin{aligned}
\left\|z_{n}-p\right\|^{2}= & \left\|T_{\delta_{n}}^{(\theta, \varphi)}\left(x_{n}-\delta_{n} F x_{n}\right)-T_{\delta_{n}}^{(\theta, \varphi)}\left(p-\delta_{n} F p\right)\right\|^{2} \\
\leq & \left\langle\left(x_{n}-\delta_{n} F x_{n}\right)-\left(p-\delta_{n} F p\right), z_{n}-p\right\rangle \\
= & \frac{1}{2}\left[\left\|\left(x_{n}-\delta_{n} F x_{n}\right)-\left(p-\delta_{n} F p\right)\right\|^{2}+\left\|z_{n}-p\right\|^{2}\right. \\
& \left.-\left\|\left(x_{n}-\delta_{n} F x_{n}\right)-\left(p-\delta_{n} F p\right)-\left(z_{n}-p\right)\right\|^{2}\right] \\
\leq & \frac{1}{2}\left[\left\|x_{n}-p\right\|^{2}+\left\|z_{n}-p\right\|^{2}-\left\|x_{n}-z_{n}-\delta_{n}\left(F x_{n}-F p\right)\right\|^{2}\right] \\
= & \frac{1}{2}\left[\left\|x_{n}-p\right\|^{2}+\left\|z_{n}-p\right\|^{2}-\left\|x_{n}-z_{n}\right\|^{2}\right. \\
& \left.+2 \delta_{n}\left\langle F x_{n}-F p, x_{n}-z_{n}\right\rangle-\delta_{n}^{2}\left\|F x_{n}-F p\right\|^{2}\right],
\end{aligned}
$$

which implies that

$$
\begin{aligned}
\left\|z_{n}-p\right\|^{2} & \leq\left\|x_{n}-p\right\|^{2}-\left\|x_{n}-z_{n}\right\|^{2}+2 \delta_{n}\left\langle F x_{n}-F p, x_{n}-z_{n}\right\rangle \\
& \leq\left\|x_{n}-p\right\|^{2}-\left\|x_{n}-z_{n}\right\|^{2}+2 \delta_{n}\left\|F x_{n}-F p\right\|\left\|x_{n}-z_{n}\right\| .
\end{aligned}
$$

From (3.7) and (3.18), we have

$$
\begin{aligned}
\left\|x_{n+1}-p\right\|^{2} \leq & \alpha_{n}\left\|\gamma f\left(x_{n}\right)-A p\right\|^{2}+\beta_{n}\left\|x_{n}-p\right\|^{2}+\left(1-\beta_{n}-\alpha_{n} \bar{\gamma}\right)\left\|y_{n}-p\right\|^{2} \\
\leq & \alpha_{n}\left\|\gamma f\left(x_{n}\right)-A p\right\|^{2}+\beta_{n}\left\|x_{n}-p\right\|^{2}+\left(1-\beta_{n}-\alpha_{n} \bar{\gamma}\right)\left\|z_{n}-p\right\|^{2} \\
\leq & \alpha_{n}\left\|\gamma f\left(x_{n}\right)-A p\right\|^{2}+\beta_{n}\left\|x_{n}-p\right\|^{2}+\left(1-\beta_{n}-\alpha_{n} \bar{\gamma}\right)\left[\left\|x_{n}-p\right\|^{2}\right. \\
& \left.-\left\|x_{n}-z_{n}\right\|^{2}+2 \delta_{n}\left\|F x_{n}-F p\right\|\left\|x_{n}-z_{n}\right\|\right] .
\end{aligned}
$$

It follows that

$$
\begin{gathered}
\left(1-\beta_{n}-\alpha_{n} \bar{\gamma}\right)\left\|x_{n}-z_{n}\right\|^{2} \leq \\
\alpha_{n}\left\|\gamma f\left(x_{n}\right)-A p\right\|^{2}+\left(\left\|x_{n}-p\right\|+\left\|x_{n+1}-p\right\|\right)\left\|x_{n}-x_{n+1}\right\| \\
+2\left(1-\beta_{n}-\alpha_{n} \bar{\gamma}\right) \delta_{n}\left\|F x_{n}-F p\right\|\left\|x_{n}-z_{n}\right\| .
\end{gathered}
$$

Since $\left\|F x_{n}-F p\right\| \rightarrow 0$ as $n \rightarrow \infty$, we obtain

$$
\lim _{n \rightarrow \infty}\left\|x_{n}-z_{n}\right\|=0 .
$$

Thus, from (3.16), (3.17), and (3.19) we obtain

$$
\begin{aligned}
\lim _{n \rightarrow \infty}\left\|z_{n}-y_{n}\right\| & =\lim _{n \rightarrow \infty}\left\|\left(z_{n}-u_{n}\right)-(p-u)+\left(u_{n}-y_{n}\right)+(p-u)\right\| \\
& \leq \lim _{n \rightarrow \infty}\left\|\left(z_{n}-u_{n}\right)-(p-u)\right\|+\lim _{n \rightarrow \infty}\left\|\left(u_{n}-y_{n}\right)+(p-u)\right\| \\
& =0
\end{aligned}
$$


and

$$
\begin{aligned}
\lim _{n \rightarrow \infty}\left\|x_{n}-y_{n}\right\| & \leq \lim _{n \rightarrow \infty}\left\|x_{n}-z_{n}\right\|+\lim _{n \rightarrow \infty}\left\|z_{n}-y_{n}\right\| \\
& =0 .
\end{aligned}
$$

Since $J_{M, \lambda}$ is 1-inverse strongly monotone, we have

$$
\begin{aligned}
\left\|w_{n}-p\right\|^{2}= & \left\|J_{M, \lambda}\left(y_{n}-\lambda B y_{n}\right)-J_{M, \lambda}(p-\lambda B p)\right\|^{2} \\
\leq & \left\langle\left(y_{n}-\lambda B y_{n}\right)-(p-\lambda B p), w_{n}-p\right\rangle \\
= & \frac{1}{2}\left[\left\|\left(y_{n}-\lambda B y_{n}\right)-(p-\lambda B p)\right\|^{2}+\left\|w_{n}-p\right\|^{2}\right. \\
& \left.-\left\|\left(y_{n}-\lambda B y_{n}\right)-(p-\lambda B p)-\left(w_{n}-p\right)\right\|^{2}\right] \\
\leq & \frac{1}{2}\left(\left\|y_{n}-p\right\|^{2}+\left\|w_{n}-p\right\|^{2}-\left\|\left(y_{n}-w_{n}\right)-\lambda\left(B y_{n}-B p\right)\right\|^{2}\right) \\
\leq & \frac{1}{2}\left(\left\|y_{n}-p\right\|^{2}+\left\|w_{n}-p\right\|^{2}-\left\|y_{n}-w_{n}\right\|^{2}+2 \lambda\left\langle y_{n}-w_{n}, B y_{n}-B p\right\rangle\right. \\
& \left.-\lambda^{2}\left\|B y_{n}-B p\right\|^{2}\right),
\end{aligned}
$$

which implies that

$$
\left\|w_{n}-p\right\|^{2} \leq\left\|y_{n}-p\right\|^{2}-\left\|y_{n}-w_{n}\right\|^{2}+2 \lambda\left\|y_{n}-w_{n}\right\|\left\|B y_{n}-B p\right\| .
$$

Substituting (3.15) into (3.20), we have

$$
\begin{aligned}
\left\|w_{n}-p\right\|^{2} \leq & \left\|x_{n}-p\right\|^{2}-\left\|\left(u_{n}-y_{n}\right)+(p-u)\right\|^{2} \\
& +2 \mu_{1}\left\|B_{1} u_{n}-B_{1} u\right\|\left\|\left(u_{n}-y_{n}\right)+(p-u)\right\| \\
& -\left\|y_{n}-w_{n}\right\|^{2}+2 \lambda\left\|y_{n}-w_{n}\right\|\left\|B y_{n}-B p\right\| .
\end{aligned}
$$

It follows from (3.21) that

$$
\begin{aligned}
& \left\|x_{n+1}-p\right\|^{2} \\
& \leq \alpha_{n}\left\|\gamma f\left(x_{n}\right)-A p\right\|^{2}+\beta_{n}\left\|x_{n}-p\right\|^{2}+\left(1-\beta_{n}-\alpha_{n} \bar{\gamma}\right)\left\|w_{n}-p\right\|^{2} \\
& \leq \alpha_{n}\left\|\gamma f\left(x_{n}\right)-A p\right\|^{2}+\left(1-\alpha_{n} \bar{\gamma}\right)\left\|x_{n}-p\right\|^{2} \\
& \quad-\left(1-\beta_{n}-\alpha_{n} \bar{\gamma}\right)\left\|\left(u_{n}-y_{n}\right)+(p-u)\right\|^{2} \\
& \quad+2 \mu_{1}\left(1-\beta_{n}-\alpha_{n} \bar{\gamma}\right)\left\|B_{1} u_{n}-B_{1} u\right\|\left\|\left(u_{n}-y_{n}\right)+(p-u)\right\| \\
& \quad-\left(1-\beta_{n}-\alpha_{n} \bar{\gamma}\right)\left\|y_{n}-w_{n}\right\|^{2}+2 \lambda\left(1-\beta_{n}-\alpha_{n} \bar{\gamma}\right)\left\|y_{n}-w_{n}\right\|\left\|B y_{n}-B p\right\|,
\end{aligned}
$$

which gives

$$
\begin{aligned}
& \left(1-\beta_{n}-\alpha_{n} \bar{\gamma}\right)\left\|y_{n}-w_{n}\right\|^{2} \\
& \quad \leq \alpha_{n}\left\|\gamma f\left(x_{n}\right)-A p\right\|^{2}+\left\|x_{n}-x_{n+1}\right\|\left(\left\|x_{n}-p\right\|+\left\|x_{n+1}-p\right\|\right)
\end{aligned}
$$




$$
\begin{aligned}
& -\left(1-\beta_{n}-\alpha_{n} \bar{\gamma}\right)\left\|\left(u_{n}-y_{n}\right)+(p-u)\right\|^{2} \\
& +2 \mu_{1}\left(1-\beta_{n}-\alpha_{n} \bar{\gamma}\right)\left\|B_{1} u_{n}-B_{1} u\right\|\left\|\left(u_{n}-y_{n}\right)+(p-u)\right\| \\
& +2 \lambda\left(1-\beta_{n}-\alpha_{n} \bar{\gamma}\right)\left\|y_{n}-w_{n}\right\|\left\|B y_{n}-B p\right\| .
\end{aligned}
$$

Since $\alpha_{n} \rightarrow 0,\left\|x_{n+1}-x_{n}\right\| \rightarrow 0,\left\|\left(u_{n}-y_{n}\right)+(p-u)\right\| \rightarrow 0,\left\|B y_{n}-B p\right\| \rightarrow 0$ as $n \rightarrow \infty$, we have $\lim _{n \rightarrow \infty}\left\|y_{n}-w_{n}\right\|=0$.

Step 5. We show $\lim _{n \rightarrow \infty}\left\|T(u) y_{n}-y_{n}\right\|=0$.

Denote $h_{n}=\frac{1}{t_{n}} \int_{0}^{t_{n}} T(u) w_{n} d u$. From (3.1), $\lim _{n \rightarrow \infty} \alpha_{n}=0$, and $\lim _{n \rightarrow \infty} \beta_{n}=0$ we have

$$
\begin{aligned}
\left\|x_{n+1}-h_{n}\right\| & =\left\|\alpha_{n} \gamma f\left(x_{n}\right)+\beta_{n} x_{n}+\left(\left(1-\beta_{n}\right) I-\alpha_{n} A\right) h_{n}-h_{n}\right\| \\
& \leq \alpha_{n}\left\|\gamma f\left(x_{n}\right)-A h_{n}\right\|+\beta_{n}\left\|x_{n}-h_{n}\right\| \\
& \rightarrow 0 \quad \text { as } n \rightarrow \infty .
\end{aligned}
$$

Let $K=\left\{w \in C:\|w-p\| \leq \frac{1}{\bar{\gamma}-\gamma \alpha}\|\gamma f(p)-A p\|\right\}$. Then $K$ is a nonempty bounded closed convex subset of $C$ which is $T(u)$-invariant for each $u \in[0, \infty)$ and contains $\left\{x_{n}\right\}$. It follows from Lemma 2.5 that

$$
\lim _{n \rightarrow \infty}\left\|h_{n}-T(u) h_{n}\right\|=0, \quad u \geq 0
$$

and from (3.22) and (3.23), we have

$$
\begin{aligned}
\left\|x_{n+1}-T(u) x_{n+1}\right\| & \leq\left\|x_{n+1}-h_{n}\right\|+\left\|h_{n}-T(u) h_{n}\right\|+\left\|T(u) h_{n}-T(u) x_{n+1}\right\| \\
& \leq 2\left\|x_{n+1}-h_{n}\right\|+\left\|h_{n}-T(u) h_{n}\right\| \\
& \rightarrow 0 \quad \text { as } n \rightarrow \infty
\end{aligned}
$$

Hence

$$
\lim _{n \rightarrow \infty}\left\|x_{n}-T(u) x_{n}\right\|=0
$$

Furthermore, from Step 4 we have for every $u \in[0, \infty)$ that

$$
\left\|T(u) y_{n}-T(u) x_{n}\right\| \leq\left\|y_{n}-x_{n}\right\| \rightarrow 0 \quad \text { as } n \rightarrow 0 .
$$

So, we obtain from (3.24)

$$
\begin{aligned}
\left\|T(u) y_{n}-x_{n}\right\| & \leq\left\|T(u) y_{n}-T(u) x_{n}\right\|+\left\|T(u) x_{n}-x_{n}\right\| \\
& \rightarrow 0 \quad \text { as } n \rightarrow \infty .
\end{aligned}
$$

Hence, we have for every $u \in[0, \infty)$ that

$$
\begin{aligned}
\left\|T(u) y_{n}-y_{n}\right\| & \leq\left\|T(u) y_{n}-x_{n}\right\|+\left\|x_{n}-y_{n}\right\| \\
& \rightarrow 0 \quad \text { as } n \rightarrow \infty
\end{aligned}
$$


Step 6. We show that $\limsup _{n \rightarrow \infty}\left\langle\gamma f\left(x^{*}\right)-A x^{*}, x_{n}-x^{*}\right\rangle \leq 0$ and $\limsup _{n \rightarrow \infty}\left\langle\gamma f\left(x^{*}\right)-\right.$ $\left.A x^{*}, \frac{1}{t_{n}} \int_{0}^{t_{n}} T(u) w_{n} d u-x^{*}\right\rangle \leq 0$, where $x^{*}=P_{\mathcal{F}}\left(\gamma f\left(x^{*}\right)+(I-A)\left(x^{*}\right)\right)$.

Indeed, take a subsequence $\left\{x_{n_{i}}\right\}$ of $\left\{x_{n}\right\}$ such that

$$
\limsup _{n \rightarrow \infty}\left\langle\gamma f\left(x^{*}\right)-A x^{*}, x_{n}-x^{*}\right\rangle=\lim _{i \rightarrow \infty}\left\langle\gamma f\left(x^{*}\right)-A x^{*}, x_{n_{i}}-x^{*}\right\rangle .
$$

Since $\left\{y_{n_{i}}\right\}$ is bounded, we can assume that $y_{n_{i}} \rightarrow w$. First, we prove that $w \in F(\Im)$.

Assume the contrary that $w \neq T(u) w$ for some $u \in[0, \infty)$. Then by Opial's condition, we obtain from Step 5 that

$$
\begin{aligned}
\liminf _{j \rightarrow \infty}\left\|y_{n_{j}}-w\right\| & <\liminf _{j \rightarrow \infty}\left\|y_{n_{j}}-T(u) w\right\| \\
& \leq \liminf _{j \rightarrow \infty}\left(\left\|y_{n_{j}}-T(u) y_{n_{j}}\right\|+\left\|T(u) y_{n_{j}}-T(u) w\right\|\right) \\
& \leq \liminf _{j \rightarrow \infty}\left\|y_{n_{j}}-w\right\| .
\end{aligned}
$$

This is a contraction. Hence, $w \in F(\Im)$.

Next, let us show that $w \in G M E P$.

From $z_{n}=T_{\delta_{n}}^{(\theta, \varphi)}\left(x_{n}-\delta_{n} F x_{n}\right)$, we obtain

$$
\theta\left(z_{n}, y\right)+\varphi(y)-\varphi\left(z_{n}\right)+\frac{1}{\delta_{n}}\left\langle y-z_{n}, z_{n}-\left(x_{n}-\delta_{n} F x_{n}\right)\right) \geq 0, \quad \forall y \in C .
$$

It follows from $(\mathrm{H} 2)$ that

$$
\varphi(y)-\varphi\left(z_{n}\right)+\frac{1}{\delta_{n}}\left\langle y-z_{n}, z_{n}-\left(x_{n}-\delta_{n} F x_{n}\right)\right| \geq \theta\left(y, z_{n}\right), \quad \forall y \in C .
$$

Replacing $n$ by $n_{i}$, we have

$$
\varphi(y)-\varphi\left(z_{n_{i}}\right)+\left\langle y-z_{n_{i}}, F x_{n_{i}}\right\rangle+\left\langle y-z_{n_{i}}, \frac{z_{n_{i}}-x_{n_{i}}}{\delta_{n_{i}}}\right\rangle \geq \theta\left(y, z_{n_{i}}\right), \quad \forall y \in C .
$$

Let $z_{t}=t y+(1-t) w$ for all $t \in[0,1]$ and $y \in C$. Then we have $z_{t} \in C$. It follows from (3.28) that

$$
\begin{aligned}
\left\langle z_{t}-z_{n_{i}}, F z_{t}\right\rangle \geq & \left\langle z_{t}-z_{n_{i}}, F z_{t}\right\rangle-\varphi\left(z_{t}\right)+\varphi\left(z_{n_{i}}\right)-\left\langle z_{t}-z_{n_{i}}, F x_{n_{i}}\right\rangle \\
& -\left\langle z_{t}-z_{n_{i}}, \frac{z_{n_{i}}-x_{n_{i}}}{\delta_{n_{i}}}\right\rangle+\theta\left(z_{t}, z_{n_{i}}\right) \\
\leq & \left\langle z_{t}-z_{n_{i}}, F z_{t}-F z_{n_{i}}\right\rangle+\left\langle z_{t}-z_{n_{i}}, F z_{n_{i}}-F x_{n_{i}}\right\rangle \\
& -\varphi\left(z_{t}\right)+\varphi\left(z_{n_{i}}\right)-\left\langle z_{t}-z_{n_{i}}, \frac{z_{n_{i}}-x_{n_{i}}}{\delta_{n_{i}}}\right\rangle+\theta\left(z_{t}, z_{n_{i}}\right) .
\end{aligned}
$$

Since $\left\|z_{n_{i}}-x_{n_{i}}\right\| \rightarrow 0$, we have $\left\|F z_{n_{i}}-F x_{n_{i}}\right\| \rightarrow 0$ as $n_{i} \rightarrow \infty$. From the monotonity of $F$, we have

$$
\left\langle F z_{t}-F z_{n_{i}}, z_{t}-z_{n_{i}}\right\rangle \geq 0
$$


From (H4), $\frac{z_{n_{i}}-x_{n_{i}}}{\delta n_{i}} \rightarrow 0$ and $z_{n_{i}} \rightarrow w$, we have

$$
\left\langle z_{t}-w, F z_{t}\right\rangle \geq-\varphi\left(z_{t}\right)+\varphi(w)+\theta\left(z_{t}, w\right)
$$

as $n_{i} \rightarrow \infty$. By (H1), (H4), and (3.29), we obtain

$$
\begin{aligned}
0 & =\theta\left(z_{t}, z_{t}\right)+\varphi\left(z_{t}\right)-\varphi\left(z_{t}\right) \\
& \leq t \theta\left(z_{t}, y\right)+(1-t) \theta\left(z_{t}, w\right)+t \varphi(y)+(1-t) \varphi(w)-\varphi\left(z_{t}\right) \\
& \leq t\left[\theta\left(z_{t}, y\right)+\varphi(y)-\varphi\left(z_{t}\right)\right]+(1-t)\left\langle z_{t}-w, F z_{t}\right\rangle \\
& =t\left[\theta\left(z_{t}, y\right)+\varphi(y)-\varphi\left(z_{t}\right)\right]+(1-t) t\left\langle y-w, F z_{t}\right\rangle .
\end{aligned}
$$

Hence we obtain

$$
0 \leq \theta\left(z_{t}, y\right)+\varphi(y)-\varphi\left(z_{t}\right)+(1-t)\left\langle y-w, F z_{t}\right\rangle .
$$

Putting $t \rightarrow 0$, we have

$$
0 \leq \theta(w, y)+\varphi(y)-\varphi(w)+\langle y-w, F w\rangle, \quad \forall y \in C .
$$

This implies that $w \in G M E P$.

Next, we prove that $w \in \Omega$.

Utilizing Lemma 2.1, we have for all $x, y \in C$

$$
\begin{aligned}
\| \Gamma(x) & -\Gamma(y) \|^{2} \\
= & \| T_{\mu_{1},\left(G_{1}, \phi\right)}\left[T_{\left.\mu_{2}, G_{2}\right)}^{\left(G_{2}\right)}\left(x-\mu_{2} B_{2} x\right)-\mu_{1} B_{1} T_{\mu_{2}}^{\left(G_{2}, \psi\right)}\left(x-\mu_{2} B_{2} x\right)\right] \\
& -T_{\mu_{1}}^{\left(G_{1}, \phi\right)}\left[T_{\mu_{2}}^{\left(G_{2}, \psi\right)}\left(y-\mu_{2} B_{2} y\right)-\mu_{1} B_{1} T_{\mu_{2}}^{\left(G_{2}, \psi\right)}\left(y-\mu_{2} B_{2} y\right)\right] \|^{2} \\
\leq & \| T_{\mu_{2}}^{\left(G_{2}, \psi\right)}\left(x-\mu_{2} B_{2} x\right)-T_{\mu_{2}}^{\left(G_{2}, \psi\right)}\left(y-\mu_{2} B_{2} y\right) \\
& -\mu_{1}\left(B_{1} T_{\mu_{2}}^{\left(G_{2}, \psi\right)}\left(x-\mu_{2} B_{2} x\right)-B_{1} T_{\mu_{2}}^{\left(G_{2}, \psi\right)}\left(y-\mu_{2} B_{2} y\right)\right) \|^{2} \\
\leq & \left\|T_{\mu_{2}}^{\left(G_{2}, \psi\right)}\left(x-\mu_{2} B_{2} x\right)-T_{\mu_{2},\left(G_{2}, \psi\right)}\left(y-\mu_{2} B_{2} y\right)\right\|^{2} \\
& +\mu_{1}\left(\mu_{1}-2 \beta_{1}\right)\left\|B_{1} T_{\mu_{2}}^{\left(G_{2}, \psi\right)}\left(x-\mu_{2} B_{2} x\right)-B_{1} T_{\mu_{2}}^{\left(G_{2}, \psi\right)}\left(y-\mu_{2} B_{2} y\right)\right\|^{2} \\
\leq & \left\|T_{\mu_{2}}^{\left(G_{2}, \psi\right)}\left(x-\mu_{2} B_{2} x\right)-T_{\mu_{2},\left(G_{2}, \psi\right)}\left(y-\mu_{2} B_{2} y\right)\right\|^{2} \\
\leq & \left\|x-y-\mu_{2}\left(B_{2} x-B_{2} y\right)\right\|^{2} \\
\leq & \|x-y\|^{2}+\mu_{2}\left(\mu_{2}-2 \beta_{2}\right)\left\|B_{2} x-B_{2} y\right\|^{2} \\
\leq & \|x-y\|^{2} .
\end{aligned}
$$

This shows that $\Gamma: C \rightarrow C$ is nonexpansive. Note that

$$
\begin{aligned}
\left\|y_{n}-\Gamma\left(y_{n}\right)\right\| & =\left\|\Gamma\left(z_{n}\right)-\Gamma\left(y_{n}\right)\right\| \\
& \leq\left\|z_{n}-y_{n}\right\| \\
& \rightarrow 0 \quad \text { as } n \rightarrow \infty .
\end{aligned}
$$

According to Lemma 2.2 and Lemma 2.3, we obtain $w \in \Omega$. 
Lastly, we show that $w \in I(B, M)$.

In fact, since $B$ is a $\beta$-inverse strongly monotone, $B$ is monotone and Lipschitz continuous mapping. It follows from Lemma 2.4 that $M+B$ is a maximal monotone mapping. Let $(v, g) \in G(M+B)$. Then $g-B(v) \in M(v)$. Since $w_{n_{i}}=J_{M, \lambda}\left(y_{n_{i}}-\lambda B y_{n_{i}}\right)$, we have $y_{n_{i}}-\lambda B y_{n_{i}} \in(I+\lambda M)\left(w_{n_{i}}\right)$, i.e., $\frac{1}{\lambda}\left(y_{n_{i}}-w_{n_{i}}-\lambda A y_{n_{i}}\right) \in M\left(w_{n_{i}}\right)$. By virtue of the maximal monotonicity of $M+B$, we have

$$
\left\langle v-w_{n_{i}}, g-B(v)-\frac{1}{\lambda}\left(y_{n_{i}}-w_{n_{i}}-\lambda B y_{n_{i}}\right)\right\rangle \geq 0
$$

and hence

$$
\begin{aligned}
\left\langle v-w_{n_{i}}, g\right\rangle \geq & \left\langle v-w_{n_{i}}, B(v)+\frac{1}{\lambda}\left(y_{n_{i}}-w_{n_{i}}-\lambda B y_{n_{i}}\right)\right\rangle \\
= & \left\langle v-w_{n_{i}}, B v-B w_{n_{i}}\right\rangle+\left\langle v-w_{n_{i}}, B w_{n_{i}}-B y_{n_{i}}\right\rangle \\
& +\left\langle v-w_{n_{i}}, \frac{1}{\lambda}\left(y_{n_{i}}-w_{n_{i}}\right)\right\rangle .
\end{aligned}
$$

It follows from $\lim _{n \rightarrow \infty}\left\|w_{n}-y_{n}\right\|=0$ that we have

$$
\lim _{n \rightarrow \infty}\left\|B w_{n}-B y_{n}\right\|=0
$$

and

$$
\begin{aligned}
& w_{n_{i}} \rightarrow w, \\
& \limsup _{n \rightarrow \infty}\left\langle v-w_{n_{i}}, g\right\rangle=\langle v-w, g\rangle \geq 0 .
\end{aligned}
$$

It follows from the maximal monotonicity of $M+B$ that $\theta \in(M+B)(w)$, that is, $w \in I(B, M)$. Therefore $\omega \in \mathcal{F}=F(\Im) \cap G M E P \cap \Omega \cap I(B, M)$.

By $x^{*}=P_{\mathcal{F}}(\gamma f+(I-A))\left(x^{*}\right)$, we obtain

$$
\begin{aligned}
\limsup _{n \rightarrow \infty}\left\langle(\gamma f-A) x^{*}, x_{n}-x^{*}\right\rangle & =\lim _{i \rightarrow \infty}\left\langle(\gamma f-A) x^{*}, x_{n_{i}}-x^{*}\right\rangle \\
& =\left\langle(\gamma f-A) x^{*}, w-x^{*}\right\rangle \\
& \leq 0
\end{aligned}
$$

and

$$
\begin{aligned}
& \limsup _{n \rightarrow \infty}\left\langle(\gamma f-A) x^{*}, \frac{1}{t_{n}} \int_{0}^{t_{n}} T(u) w_{n} d u-x^{*}\right\rangle \\
& \quad=\lim _{i \rightarrow \infty}\left\langle(\gamma f-A) x^{*}, \frac{1}{t_{n_{i}}} \int_{0}^{t_{n_{i}}} T(u) w_{n_{i}} d u-x^{*}\right\rangle \\
& \quad=\left\langle(\gamma f-A) x^{*}, w-x^{*}\right\rangle \\
& \quad \leq 0,
\end{aligned}
$$

as required. 
Step 7. We prove $x_{n} \rightarrow x^{*}$ as $n \rightarrow \infty$.

By using (3.1), we have

$$
\begin{aligned}
&\left\|x_{n+1}-x^{*}\right\|^{2} \\
&=\| \alpha_{n}\left(\gamma f\left(x_{n}\right)-A x^{*}\right)+\beta_{n}\left(x_{n}-x^{*}\right) \\
&+\left(\left(1-\beta_{n}\right) I-\alpha_{n} A\right)\left(\frac{1}{t_{n}} \int_{0}^{t_{n}} T(u) w_{n} d u-x^{*}\right) \|^{2} \\
& \leq \alpha_{n}^{2}\left\|\gamma f\left(x_{n}\right)-A x^{*}\right\|^{2} \\
&+\left\|\beta_{n}\left(x_{n}-x^{*}\right)+\left(\left(1-\beta_{n}\right) I-\alpha_{n} A\right)\left(\frac{1}{t_{n}} \int_{0}^{t_{n}} T(u) w_{n} d u-x^{*}\right)\right\|^{2} \\
&+2 \alpha_{n}\left\langle\gamma f\left(x_{n}\right)-A x^{*}, \beta_{n}\left(x_{n}-x^{*}\right)+\left(\left(1-\beta_{n}\right) I-\alpha_{n} A\right)\left(\frac{1}{t_{n}} \int_{0}^{t_{n}} T(u) w_{n} d u-x^{*}\right)\right\rangle \\
& \leq \alpha_{n}^{2}\left\|\gamma f\left(x_{n}\right)-A x^{*}\right\|^{2}+\beta_{n}^{2}\left\|x_{n}-x^{*}\right\|^{2}+\left(1-\beta_{n}-\alpha_{n} \bar{\gamma}\right)^{2}\left\|w_{n}-x^{*}\right\|^{2} \\
&+2 \beta_{n}\left\langle x_{n}-x^{*},\left(\left(1-\beta_{n}\right) I-\alpha_{n} A\right)\left(\frac{1}{t_{n}} \int_{0}^{t_{n}} T(u) w_{n} d u-x^{*}\right)\right\rangle \\
&+2 \alpha_{n} \beta_{n}\left\langle\gamma f\left(x_{n}\right)-\gamma f\left(x^{*}\right), x_{n}-x^{*}\right\rangle+2 \alpha_{n} \beta_{n}\left\langle\gamma f\left(x^{*}\right)-A x^{*}, x_{n}-x^{*}\right\rangle \\
&+2 \alpha_{n}\left(1-\beta_{n}-\alpha_{n} \bar{\gamma}\right)\left\langle\gamma f\left(x_{n}\right)-\gamma f\left(x^{*}\right), \frac{1}{t_{n}} \int_{0}^{t_{n}} T(u) w_{n} d u-x^{*}\right\rangle \\
&+2 \alpha_{n}\left(1-\beta_{n}-\alpha_{n} \bar{\gamma}\right)\left\langle\gamma f\left(x^{*}\right)-A x^{*}, \frac{1}{t_{n}} \int_{0}^{t_{n}} T(u) w_{n} d u-x^{*}\right\rangle .
\end{aligned}
$$

It follows from (3.4), (3.6), and (3.7) that

$$
\begin{aligned}
\| x_{n+1} & -x^{*} \|^{2} \\
\leq & \alpha_{n}^{2}\left\|\gamma f\left(x_{n}\right)-A x^{*}\right\|^{2}+\beta_{n}^{2}\left\|x_{n}-x^{*}\right\|^{2}+\left(1-\beta_{n}-\alpha_{n} \bar{\gamma}\right)^{2}\left\|x_{n}-x^{*}\right\|^{2} \\
& +2 \beta_{n}\left(1-\beta_{n}-\alpha_{n} \bar{\gamma}\right)\left\|x_{n}-x^{*}\right\|^{2} \\
& +2 \alpha_{n} \beta_{n} \gamma \alpha\left\|x_{n}-x^{*}\right\|^{2}+2 \alpha_{n} \beta_{n}\left\langle\gamma f\left(x^{*}\right)-A x^{*}, x_{n}-x^{*}\right\rangle \\
& +2 \alpha_{n}\left(1-\beta_{n}-\alpha_{n} \bar{\gamma}\right) \gamma \alpha\left\|x_{n}-x^{*}\right\|^{2} \\
& +2 \alpha_{n}\left(1-\beta_{n}-\alpha_{n} \bar{\gamma}\right)\left\langle\gamma f\left(x^{*}\right)-A x^{*}, \frac{1}{t_{n}} \int_{0}^{t_{n}} T(u) w_{n} d u-x^{*}\right\rangle \\
\leq & {\left[1-2 \alpha_{n}\left(\bar{\gamma}-\gamma \alpha+\alpha_{n} \bar{\gamma} \gamma \alpha\right)\right]\left\|x_{n}-x^{*}\right\|^{2}+\alpha_{n}^{2}\left\|\gamma f\left(x_{n}\right)-A x^{*}\right\|^{2} } \\
& +2 \alpha_{n} \beta_{n}\left\langle\gamma f\left(x^{*}\right)-A x^{*}, x_{n}-x^{*}\right\rangle \\
& +2 \alpha_{n}\left(1-\beta_{n}-\alpha_{n} \bar{\gamma}\right)\left\langle\gamma f\left(x^{*}\right)-A x^{*}, \frac{1}{t_{n}} \int_{0}^{t_{n}} T(u) w_{n} d u-x^{*}\right\rangle \\
& +\alpha_{n}^{2} \bar{\gamma}^{2}\left\|x_{n}-x^{*}\right\|^{2} \\
= & \left(1-\bar{\alpha}_{n}\right)\left\|x_{n}-x^{*}\right\|^{2}+\bar{\beta}_{n},
\end{aligned}
$$


where

$$
\begin{aligned}
\bar{\alpha}_{n}= & 2 \alpha_{n}\left(\bar{\gamma}-\gamma \alpha+\alpha_{n} \bar{\gamma} \gamma \alpha\right), \\
\bar{\beta}_{n}= & \alpha_{n}^{2}\left\|\gamma f\left(x_{n}\right)-A x^{*}\right\|^{2}+2 \alpha_{n} \beta_{n}\left(\gamma f\left(x^{*}\right)-A x^{*}, x_{n}-x^{*}\right\rangle \\
& +2 \alpha_{n}\left(1-\beta_{n}-\alpha_{n} \bar{\gamma}\right)\left\langle\gamma f\left(x^{*}\right)-A x^{*}, \frac{1}{t_{n}} \int_{0}^{t_{n}} T(u) w_{n} d u-x^{*}\right\rangle \\
& +\alpha_{n}^{2} \bar{\gamma}^{2}\left\|x_{n}-x^{*}\right\|^{2} .
\end{aligned}
$$

It is easily seen that $\bar{\alpha}_{n} \rightarrow 0$ as $n \rightarrow \infty, \sum_{n=1}^{\infty} \bar{\alpha}_{n}=+\infty$ and $\lim \sup _{n \rightarrow \infty} \frac{\bar{\beta}_{n}}{\bar{\alpha}_{n}} \leq 0$. Hence, applying Lemma 2.6 we immediately obtain $x_{n} \rightarrow x^{*}$ as $n \rightarrow \infty$. This completes the proof.

Remark 3.1 Let us consider the following sequences:

$$
\alpha_{n}=\frac{1}{n^{\frac{1}{4}}}, \quad \beta_{n}=\frac{1}{n^{2}}, \quad \delta_{n}=\frac{1}{n^{\frac{1}{4}}}+\frac{1}{2} \quad \text { and } \quad t_{n}=n, \quad \forall n \geq 1 .
$$

It is easy to see that all hypotheses (C1)-(C4) of Theorem 3.1 are satisfied.

By Theorem 3.1, we can obtain the following results immediately.

Corollary 3.1 Let $C$ be a nonempty closed convex subset of a real Hilbert space H. Let $\theta, G_{1}, G_{2}: C \times C \rightarrow \mathbb{R}$ be three bifunctions which satisfy assumptions (H1)-(H4) and $\varphi, \phi, \psi: C \rightarrow \mathbb{R}$ be three lower semicontinuous and convex functions with restriction (A1) or (A2). Let $B, B_{1}, B_{2}: C \rightarrow H$ be $\beta$-inverse strongly monotone, $\beta_{1}$-inverse strongly monotone and $\beta_{2}$-inverse strongly monotone, respectively and $M: H \rightarrow 2^{H}$ be a maximal monotone mapping. Let $\Im=\{T(u): 0 \leq u<\infty\}$ be a one-parameter nonexpansive semigroup on $H$ such that $\mathcal{F}_{1}=F(\Im) \cap M E P \cap \Omega \cap I(B, M) \neq \phi$. Let $\left\{t_{n}\right\} \subset(0, \infty)$ be a real sequence such that $\lim _{n \rightarrow \infty} t_{n}=\infty$. Let $f$ be a contraction from $C$ into itself with a constant $\alpha(0<\alpha<1)$ and let $A$ be a strongly positive linear bounded operator with coefficient $\bar{\gamma}>0$ such that $\|A\| \leq 1$. Assume that $0<\gamma<\frac{\bar{\gamma}}{\alpha\left(1-\alpha_{n} \bar{\gamma}\right)}$. Let $x_{1} \in C$ and let $\left\{x_{n}\right\}$ be a sequence defined by

$$
\left\{\begin{aligned}
\theta\left(z_{n}, y\right)+\varphi(y)-\varphi\left(z_{n}\right)+\frac{1}{\delta_{n}}\left\langle y-z_{n}, z_{n}-x_{n}\right\rangle \geq 0, \quad \forall y \in C, \\
y_{n}=T_{\mu_{1}}^{\left(G_{1}, \phi\right)}\left[T_{\mu_{2}}^{\left(G_{2}, \psi\right)}\left(z_{n}-\mu_{2} B_{2} z_{n}\right)-\mu_{1} B_{1} T_{\mu_{2}}^{\left(G_{2}, \psi\right)}\left(z_{n}-\mu_{2} B_{2} z_{n}\right)\right], \\
x_{n+1}=\alpha_{n} \gamma f\left(x_{n}\right)+\beta_{n} x_{n} \\
\quad+\left(\left(1-\beta_{n}\right) I-\alpha_{n} A\right)\left[\frac{1}{t_{n}} \int_{0}^{t_{n}} T(u)\left(J_{M, \lambda}\left(y_{n}-\lambda B y_{n}\right)\right)\right] d u, \quad \forall n \geq 1,
\end{aligned}\right.
$$

where $\alpha_{n} \in(0,1), \mu_{1} \in\left(0,2 \beta_{1}\right], \mu_{2} \in\left(0,2 \beta_{2}\right], \lambda \in(0,2 \beta], \delta_{n} \in(0,2 \zeta]$ satisfy the following conditions:

(i) $\lim _{n \rightarrow \infty} \alpha_{n}=0, \sum_{n=1}^{\infty} \alpha_{n}=\infty$ and $\sum_{n=1}^{\infty}\left|\alpha_{n+1}-\alpha_{n}\right|<\infty$;

(ii) $\sum_{n=1}^{\infty} \beta_{n}<\infty$ and $\sum_{n=1}^{\infty}\left|\beta_{n+1}-\beta_{n}\right|<\infty$;

(iii) $\liminf _{n \rightarrow \infty} \delta_{n}>0$ and $\sum_{n=1}^{\infty}\left|\delta_{n+1}-\delta_{n}\right|<\infty$;

(iv) $\lim _{n \rightarrow \infty} \frac{\left|t_{n}-t_{n-1}\right|}{t_{n}} \frac{1}{\alpha_{n}}=0$. 
Then $\left\{x_{n}\right\}$ converges strongly to $x^{*}=P_{\mathcal{F}_{1}}(\gamma f+(I-A))\left(x^{*}\right)$, which solves the following variational inequality:

$$
\left\langle(A-\gamma f) x^{*}, x-x^{*}\right\rangle \geq 0, \quad \forall x \in \mathcal{F}_{1}
$$

and $\left(x^{*}, y^{*}\right)$ is a solution of problem $(1.5)$, where $y^{*}=T_{\mu_{2}}^{\left(G_{2}, \psi\right)}\left(x^{*}-\mu_{2} B_{2} x^{*}\right)$.

Proof In Theorem 3.1, for all $n \geq 0, z_{n}=T_{\delta_{n}}^{(\theta, \varphi)}\left(x_{n}-\delta_{n} F x_{n}\right)$ is equivalent to

$$
\theta\left(z_{n}, y\right)+\varphi(y)-\varphi\left(z_{n}\right)+\left\langle F x_{n}, y-z_{n}\right\rangle+\frac{1}{\delta_{n}}\left\langle y-z_{n}, z_{n}-x_{n}\right\rangle \geq 0, \quad \forall y \in C .
$$

Putting $F=0$, we obtain

$$
\theta\left(z_{n}, y\right)+\varphi(y)-\varphi\left(z_{n}\right)+\frac{1}{\delta_{n}}\left\langle y-z_{n}, z_{n}-x_{n}\right\rangle \geq 0, \quad \forall y \in C .
$$

By Theorem 3.1, we can easily get the desired conclusion.

Corollary 3.2 Let $C$ be a nonempty closed convex subset of a real Hilbert space $H$. Let $G_{1}, G_{2}: C \times C \rightarrow \mathbb{R}$ be two bifunctions which satisfy assumptions (H1)-(H4) and $\phi, \psi: C \rightarrow \mathbb{R}$ be two lower semicontinuous and convex functions with restriction (A1) or (A2). Let $F, B, B_{1}, B_{2}: C \rightarrow H$ be $\zeta$-inverse strongly monotone, $\beta$-inverse strongly monotone, $\beta_{1}$-inverse strongly monotone and $\beta_{2}$-inverse strongly monotone, respectively, and let $M: H \rightarrow 2^{H}$ be a maximal monotone mapping. Let $\mathfrak{s}=\{T(u): 0 \leq u<\infty\}$ be a oneparameter nonexpansive semigroup on $H$ such that $\mathcal{F}_{2}=F(\Im) \cap V I(F, C) \cap \Omega \cap I(B, M) \neq \phi$. Let $\left\{t_{n}\right\} \subset(0, \infty)$ be a real sequence such that $\lim _{n \rightarrow \infty} t_{n}=\infty$. Let $f$ be a contraction from $C$ into itself with a constant $\alpha(0<\alpha<1)$ and let $A$ be a strongly positive linear bounded operator with coefficient $\bar{\gamma}>0$ such that $\|A\| \leq 1$. Assume that $0<\gamma<\frac{\bar{\gamma}}{\alpha\left(1-\alpha_{n} \bar{\gamma}\right)}$. Let $x_{1} \in C$ and let $\left\{x_{n}\right\}$ be a sequence defined by

$$
\left\{\begin{aligned}
z_{n}= & P_{C}\left(x_{n}-\delta_{n} F x_{n}\right), \\
y_{n}= & T_{\mu_{1}}^{\left(G_{1}, \phi\right)}\left[T_{\mu_{2}}^{\left(G_{2}, \psi\right)}\left(z_{n}-\mu_{2} B_{2} z_{n}\right)-\mu_{1} B_{1} T_{\mu_{2}}^{\left(G_{2}, \psi\right)}\left(z_{n}-\mu_{2} B_{2} z_{n}\right)\right], \\
x_{n+1}= & \alpha_{n} \gamma f\left(x_{n}\right)+\beta_{n} x_{n} \\
& \quad\left(\left(1-\beta_{n}\right) I-\alpha_{n} A\right)\left[\frac{1}{t_{n}} \int_{0}^{t_{n}} T(u)\left(J_{M, \lambda}\left(y_{n}-\lambda B y_{n}\right)\right) d u\right], \quad \forall n \geq 1,
\end{aligned}\right.
$$

where $\alpha_{n} \in[0,1], \mu_{1} \in\left(0,2 \beta_{1}\right], \mu_{2} \in\left(0,2 \beta_{2}\right], \lambda \in(0,2 \beta], \delta_{n} \in(0,2 \zeta]$ satisfy the following conditions:

(i) $\lim _{n \rightarrow \infty} \alpha_{n}=0, \sum_{n=1}^{\infty} \alpha_{n}=\infty$ and $\sum_{n=1}^{\infty}\left|\alpha_{n+1}-\alpha_{n}\right|<\infty$;

(ii) $\sum_{n=1}^{\infty} \beta_{n}<\infty$ and $\sum_{n=1}^{\infty}\left|\beta_{n+1}-\beta_{n}\right|<\infty$;

(iii) $\liminf _{n \rightarrow \infty} \delta_{n}>0$ and $\sum_{n=1}^{\infty}\left|\delta_{n+1}-\delta_{n}\right|<\infty$;

(iv) $\lim _{n \rightarrow \infty} \frac{\left|t_{n}-t_{n-1}\right|}{t_{n}} \frac{1}{\alpha_{n}}=0$.

Then $\left\{x_{n}\right\}$ converges strongly to $x^{*}=P_{\mathcal{F}_{2}}(\gamma f+(I-A))\left(x^{*}\right)$, which solves the following variational inequality:

$$
\left\langle(A-\gamma f) x^{*}, x-x^{*}\right\rangle \geq 0, \quad \forall x \in \mathcal{F}_{2}
$$

and $\left(x^{*}, y^{*}\right)$ is a solution of problem $(1.5)$, where $y^{*}=T_{\mu_{2}}^{\left(G_{2}, \psi\right)}\left(x^{*}-\mu_{2} B_{2} x^{*}\right)$. 
Proof Put $\theta=0$ and $\varphi=0$ in Theorem 3.1. Then we have from (3.30)

$$
\left\langle F x_{n}, y-z_{n}\right\rangle+\frac{1}{\delta_{n}}\left\langle y-z_{n}, z_{n}-x_{n}\right\rangle \geq 0, \quad \forall y \in C, n \geq 1 .
$$

That is,

$$
\left\langle y-z_{n}, x_{n}-\delta_{n} F x_{n}-z_{n}\right\rangle \leq 0, \quad \forall y \in C
$$

It follows that $P_{C}\left(x_{n}-\delta_{n} F x_{n}\right)=z_{n}$ for all $n \geq 1$. We easily obtain the desired conclusion.

Corollary 3.3 Let $C$ be a nonempty closed convex subset of a real Hilbert space H. Let $\theta, G_{1}, G_{2}: C \times C \rightarrow \mathbb{R}$ be three bifunctions which satisfy assumptions (H1)-(H4) and $\varphi, \phi, \psi: C \rightarrow \mathbb{R}$ be three lower semicontinuous and convex functions with restriction (A1) or (A2). Let $F, B, B_{1}, B_{2}: C \rightarrow H$ be $\zeta$-inverse strongly monotone, $\beta$-inverse strongly monotone, $\beta_{1}$-inverse strongly monotone, and $\beta_{2}$-inverse strongly monotone, respectively. Let $\mathfrak{I}=\{T(u): 0 \leq u<\infty\}$ be a one-parameter nonexpansive semigroup on $H$ such that $\mathcal{F}_{3}=F(\Im) \cap G M E P \cap \Omega \cap V I(F, C) \neq \phi$. Let $\left\{t_{n}\right\} \subset(0, \infty)$ be a real sequence such that $\lim _{n \rightarrow \infty} t_{n}=\infty$. Let $f$ be a contraction from $C$ into itself with a constant $\alpha(0<\alpha<1)$ and let $A$ be a strongly positive linear bounded operator with coefficient $\bar{\gamma}>0$ such that $\|A\| \leq 1$. Assume that $0<\gamma<\frac{\bar{\gamma}}{\alpha\left(1-\alpha_{n} \bar{\gamma}\right)}$. Let $x_{1} \in C$ and let $\left\{x_{n}\right\}$ be a sequence defined by

$$
\left\{\begin{aligned}
z_{n}= & T_{\delta_{n}}^{(\theta, \varphi)}\left(x_{n}-\delta_{n} F x_{n}\right) \\
y_{n}= & T_{\mu_{1}}^{\left(G_{1}, \phi\right)}\left[T_{\mu_{2}}^{\left(G_{2}, \psi\right)}\left(z_{n}-\mu_{2} B_{2} z_{n}\right)-\mu_{1} B_{1} T_{\mu_{2}}^{\left(G_{2}, \psi\right)}\left(z_{n}-\mu_{2} B_{2} z_{n}\right)\right] \\
x_{n+1}= & \alpha_{n} \gamma f\left(x_{n}\right)+\beta_{n} x_{n} \\
& \quad+\left(\left(1-\beta_{n}\right) I-\alpha_{n} A\right)\left[\frac{1}{t_{n}} \int_{0}^{t_{n}} T(u)\left(P_{C}\left(y_{n}-\lambda B y_{n}\right)\right) d u\right], \quad n \geq 1
\end{aligned}\right.
$$

where $\alpha_{n} \in(0,1), \mu_{1} \in\left(0,2 \beta_{1}\right], \mu_{2} \in\left(0,2 \beta_{2}\right], \lambda \in(0,2 \beta], \delta_{n} \in(0,2 \zeta]$ satisfy the conditions (C1)-(C4). Then $\left\{x_{n}\right\}$ converges strongly to $x^{*}=P_{\mathcal{F}_{3}}(\gamma f+(I-A))\left(x^{*}\right)$, which solves the following variational inequality:

$$
\left\langle(A-\gamma f) x^{*}, x-x^{*}\right\rangle \geq 0, \quad \forall x \in \mathcal{F}_{3}
$$

and $\left(x^{*}, y^{*}\right)$ is a solution of problem $(1.5)$, where $y^{*}=T_{\mu_{2}}^{\left(G_{2}, \psi\right)}\left(x^{*}-\mu_{2} B_{2} x^{*}\right)$.

Proof Taking $J_{M, \lambda}=P_{C}$ in Theorem 3.1, we can obtain desired conclusion immediately.

Remark 3.2 Theorem 3.1 generalizes and improves Theorem 3.1 of Kumam and Wattanawitoon [14], Theorem 3.3 of Plubtieng and Punpaeng [10] and Theorem 3.1 of Shehu [13] in the following aspects:

(1) Algorithm of Theorem 3.1 is different from algorithms in [10, 13, 14].

(2) Theorem 3.1 includes Theorem 3.3 of Plubtieng and Punpaeng [10] as a special case.

(3) Theorem 3.1 improves Theorem 3.1 of Kumam and Wattanawitoon [14] since the generalized equilibrium problem that is within [14] is extended to the generalized mixed equilibrium problem. 


\section{Competing interests}

The author declares that he has no competing interests.

Received: 3 December 2013 Accepted: 25 February 2014 Published: 05 Mar 2014

\section{References}

1. Chamnarnpan, T, Kumam, P: Iterative algorithms for solving the system of mixed equilibrium problems, fixed point problems, and variational inclusions with application to minimization problem. J. Appl. Math. 29, Article ID 538912 (2012)

2. Hao, Y: Some results of variational inclusion problems and fixed point problems with applications. Appl. Math. Mech. 30, 1589-1596 (2009)

3. Jitpeera, T, Kumam, P: Hybrid algorithms for minimization problems over the solutions of generalized mixed equilibrium and variational inclusion problems. Math. Probl. Eng. 2011, Article ID 648617 (2011)

4. Bréziz, H: Operateur maximaux monotone. Mathematics Studies, vol. 5. North-Holland, Amsterdam (1973)

5. Peng, JW, Yao, JC: A new hybrid extragradient method for generalized mixed equilibrium problems, fixed point problems, and variational inequality problems. Taiwan. J. Math. 12, 1401-1432 (2008)

6. Ceng, LC, Yao, JC: A relaxed extragradient-like method for a generalized mixed equilibrium problem, a general system of generalized equilibria and a fixed point problem. Nonlinear Anal. 72, 1922-1937 (2010)

7. Takahashi, S, Takahashi, W: Strong convergence theorem for a generalized equilibrium problem and a nonexpansive mapping in a Hilbert space. Nonlinear Anal. 69, 1025-1033 (2008)

8. Blum, E, Oettli, W: From optimization and variational inequalities to equilibrium problems. Math. Stud. 63, 123-145 (1994)

9. Ceng, LC, Yao, JC: A relaxed extragradient-like method for a generalized mixed equilibrium problem, a general system of generalized equilibria and a fixed point problem. Nonlinear Anal. 72, 1922-1937 (2010)

10. Plubtieng, S, Punpaeng, R: Fixed point solutions of variational inequalities for nonexpansive semigroups in Hilbert spaces. Math. Comput. Model. 48, 279-286 (2008)

11. Ceng, LC, Wang, CY, Yao, JC: Strong convergence theorems by a relaxed extragradient method for a general system of variational inequalities. Math. Methods Oper. Res. 67, 375-390 (2008)

12. Verma, RU: On a new system of nonlinear variational inequalities and associated iterative algorithms. Math. Sci. Res. Hot-Line 3, 65-68 (1999)

13. Shehu, Y: An iterative method for nonexpansive semigroups, variational inclusions and generalized equilibrium problems. Math. Comput. Model. 55, 1301-1314 (2012)

14. Kumam, $\mathrm{P}$, Wattanawitoon, $\mathrm{K}$ : A general composite explicit iterative scheme of fixed point solutions of variational inequalities for nonexpansive semigroups. Math. Comput. Model. 53, 998-1006 (2011)

15. Li, S, Li, L, Su, Y: General iterative methods for a one-parameter nonexpansive semigroup in Hilbert spaces. Nonlinear Anal. 70, 3065-3071 (2009)

16. Plubtieng, S, Wangkeeree, R: A general viscosity approximation method of fixed point solutions of variational inequalities for nonexpansive semigroups in Hilbert spaces. Bull. Korean Math. Soc. 45, 717-728 (2008)

17. Opial, Z: Weak convergence of the sequence of successive approximations for nonexpansive mappings. Bull. Am. Math. Soc. 73, 595-597 (1967)

18. Goebel, K, Kirk, WA: Topics on Metric Fixed Point Theory. Cambridge University Press, Cambridge (1990)

19. Shimizu, T, Takahashi, W: Strong convergence of common fixed points of families of nonexpansive mappings. J. Math. Anal. Appl. 211, 71-83 (1997)

10.1186/1687-1812-2014-57

Cite this article as: Jeong: Fixed point solutions of generalized mixed equilibrium problems and variational inclusion problems for nonexpansive semigroups. Fixed Point Theory and Applications 2014, 2014:57

\section{Submit your manuscript to a SpringerOpen ${ }^{\circ}$ journal and benefit from:}

- Convenient online submission

Rigorous peer review

- Immediate publication on acceptance

Open access: articles freely available online

- High visibility within the field

- Retaining the copyright to your article 